TRANSACTIONS OF THE

AMERICAN MATHEMATICAL SOCIETY

Volume 359, Number 5, May 2007, Pages 2421-2441

S 0002-9947(06)04267-X

Article electronically published on December 5, 2006

\title{
RAPID DECAY OF CORRELATIONS FOR NONUNIFORMLY HYPERBOLIC FLOWS
}

\author{
IAN MELBOURNE
}

\begin{abstract}
We show that superpolynomial decay of correlations (rapid mixing) is prevalent for a class of nonuniformly hyperbolic flows. These flows are the continuous time analogue of the class of nonuniformly hyperbolic maps for which Young proved exponential decay of correlations. The proof combines techniques of Dolgopyat and operator renewal theory.

It follows from our results that planar periodic Lorentz flows with finite horizons and flows near homoclinic tangencies are typically rapid mixing.
\end{abstract}

\section{INTRODUCTION}

Let $(M, \nu)$ be a probability space. Given a measure preserving flow $\phi_{t}: M \rightarrow$ $M$ and observables $v, w \in L^{2}(M)$, we define the correlation function $\rho_{v, w}(t)=$ $\int_{M} v w \circ \phi_{t} d \nu-\int_{M} v d \nu \int_{M} w d \nu$. The flow is mixing if $\lim _{t \rightarrow \infty} \rho_{v, w}(t)=0$ for all $v, w \in L^{2}(M)$.

Of interest is the rate of decay of correlations, namely the rate at which $\rho_{v, w}(t)$ converges to zero. For nontrivial mixing flows, the decay rate is arbitrarily slow for $L^{2}$ observables. Hence the aim is to establish decay rates under regularity hypotheses on the flow $\phi_{t}$, the measure $\nu$, and the observables $v, w$.

Suppose that $\Lambda \subset M$ is a uniformly hyperbolic (Axiom A) basic set for a smooth flow $\phi_{t}: M \rightarrow M$ and that $\nu$ is an equilibrium state for a Hölder potential [7. If $\Lambda$ is mixing, then Bowen and Ruelle [7] asked whether $\Lambda$ has exponential decay of correlations $\left(\rho_{v, w}(t)=O\left(e^{-c t}\right)\right.$ for some $\left.c>0\right)$ for sufficiently regular $v, w$. (In the discrete time case, it is well-known that Axiom A diffeomorphisms enjoy exponential decay of correlations.) Until recently, exponential decay was established only for Anosov flows with additional algebraic structure. Moreover, Ruelle 30] showed that mixing Axiom A flows need not have exponential decay of correlations, and Pollicott [28] showed that the decay rates could be arbitrarily slow.

In 1998, Dolgopyat [17] (building upon results of Chernov [12) showed that geodesic flows on surfaces of negative curvature have exponential decay of correlations for Hölder observables. Liverani 23] extended this result to arbitrary dimensional geodesic flows in negative curvature and more generally to contact Anosov flows.

Received by the editors January 31, 2005 and, in revised form, July 14, 2005.

2000 Mathematics Subject Classification. Primary 37A25, 37D25, 37D50.

This research was supported in part by EPSRC Grant GR/S11862/01. Technological support by the University of Houston is gratefully acknowledged.

(C)2006 American Mathematical Society Reverts to public domain 28 years from publication 
Pollicott 29] proved exponential decay for a class of uniformly hyperbolic attractors with one-dimensional unstable manifolds. However, it remains an open question whether exponential decay of correlations is typical in any reasonable sense for Axiom A (even Anosov) flows.

Dolgopyat 18 introduced the weaker notion of rapid mixing (superpolynomial decay of correlations) where for any $n \geq 1, \rho_{v, w}(t)=O\left(t^{-n}\right)$ for sufficiently regular observables, and showed that rapid mixing is 'prevalent': it suffices that the flow contains two periodic solutions with periods whose ratio is Diophantine. In addition, Dolgopyat [17 showed that for Anosov flows, joint nonintegrability of the stable and unstable foliations (an open and dense condition by methods of Brin [8, 9]) implies rapid mixing. Field, Melbourne and Török [19] introduced new techniques and combined them with Dolgopyat's work to prove that amongst $C^{r}$ Axiom A flows, $r \geq 2$, an open and dense set of flows is rapid mixing, with uniform implied constants - stable rapid mixing. (In [15, this result is misattributed to Dolgopyat.)

Rapid mixing for nonuniformly hyperbolic flows. Parallel to the advances for uniformly hyperbolic flows above, Young [35] established exponential decay of correlations for a class of nonuniformly hyperbolic maps including billiards and Hénon-like maps 4]. In this paper, we use operator renewal theory 31, 20] to extend the ideas of Dolgopyat 18 to a large class of nonuniformly hyperbolic flows, namely the continuous time analogue of the nonuniformly hyperbolic maps studied in Young [35]. Roughly speaking, the main result of this paper is that

\section{A 'prevalent' set of nonuniformly hyperbolic flows are rapid mixing.}

Again, rapid mixing is established for sufficiently regular observables, and prevalence is understood in the sense that a Diophantine condition on finitely many periods is sufficient to guarantee rapid mixing.

Limit laws for time-one maps of nonuniformly hyperbolic flows. A simple consequence of our main result, following [25], is that the (functional) central limit theorem holds for the time-one map of a typical nonuniformly hyperbolic flow. (The central limit theorem for the flow itself is a weaker property and holds regardless of rapid mixing [26].) A stronger result than the central limit theorem is the almost sure invariance principle. This is known for nonuniformly hyperbolic flows 24] and a natural question is to establish this (at least typically) for their time-one maps. The methods in 24, 25] do not seem to resolve this issue.

Lorentz gases. See [15] for a survey of results about Lorentz gases. The planar periodic Lorentz gas is a class of examples introduced by Sinai 32 . The Lorentz flow is a billiard flow on $\mathbb{T}^{2}-\Omega$ where $\Omega$ is a disjoint union of convex regions with $C^{3}$ boundaries. (The phase-space of the flow is three-dimensional; planar position and direction.) The flow has a natural global cross-section $M=\partial \Omega \times[-\pi / 2, \pi / 2]$ corresponding to collisions, and the Poincaré map $T: M \rightarrow M$ is called the billiard map. Bunimovich, Sinal and Chernov [11] proved stretched exponential decay rates for the billiard map and exponential decay rates were established by Young [35. Denote the return time function by $h: M \rightarrow \mathbb{R}^{+}$. The Lorentz flow satisfies the finite horizon condition if $h$ is uniformly bounded. It is strongly conjectured that exponential decay of correlations holds for the Lorentz flow with finite horizons, 
but previously no results on the rate of decay were available. It follows from our main result that

A prevalent set of planar periodic Lorentz flows with finite horizon are rapid mixing.

Once again, we emphasize that rapid mixing is proved for observables which are smooth along the the flow (which is not the case for position or velocity). Consequences of this result include the central limit theorem for the time-one map of a typical planar periodic Lorentz flow with finite horizon. (The central limit theorem and almost sure invariance principle are always satisfied by the flows themselves [11, 24].)

Our results also apply to externally forced periodic Lorentz gases and to planar dispersing billiards on a table whose sides are convex inwards, under the hypothesis that the corner points have positive angles. (The corresponding billiard maps were studied by Chernov in [14] and [13] respectively, and have exponential decay of correlations. In the case of the billiards with corners, a technical assumption (condition $\left(^{*}\right)$ in [15, Section 5.1, paragraph B]) is required.)

Flows near homoclinic tangencies. Benedicks and Carleson [2] studied the Hénon map $T_{a, b}(x, y)=\left(1-a x^{2}+y, b x\right)$ and proved the existence of a strange attractor for a positive measure of parameters $a, b$. The attractor admits an SRB measure [3] and was shown to have exponential decay of correlations by Benedicks and Young [4].

Mora and Viana [27] showed that Hénon-like attractors arise for positive measure sets of parameters in the unfoldings of homoclinic tangencies for surface diffeomorphisms, and these results were extended to higher dimensions by [34, 16].

The above results combined with those in this paper show that a positive measure set of flows near a homoclinic tangency are rapid mixing.

. The above examples can be viewed as suspension flows over a nonuniformly hyperbolic map $T: M \rightarrow M$. In general, we do not require a global cross-section $M$. It suffices that the flow can be modelled by a suspension of a nonuniformly hyperbolic map (in the same way that a hyperbolic basic set for an Axiom A flow is modelled by a suspension of a uniformly hyperbolic map [5]).

Remark 1.1. Two natural directions in which our results might be extended are:

(1) The class of nonuniformly hyperbolic maps studied by Young 35] possess exponential decay of correlations, and we prove rapid mixing for the analogous class of flows in this paper. In a subsequent paper, Young [36] introduces a more general class of nonuniformly hyperbolic maps with subexponential decay of correlations. Presently, we have no results for the corresponding class of flows.

(2) Our boundedness assumption on the roof function $h$ excludes an important class of flows known as singular hyperbolic flows (including geometric Lorenz attractors) for which $h$ has a logarithmic singularity. It is plausible that the techniques in this paper apply to such flows, and this will be the subject of future work. (In the present paper, the boundedness assumption is relaxed in Section 3.)

The remainder of the paper is organised as follows. In Section 2, we state our results on rapid mixing, first for nonuniformly expanding semiflows, and then for nonuniformly hyperbolic flows. Nonuniformly expanding maps have an induced return map that is Gibbs-Markov [1], and in Section 3 we study rapid mixing for suspension semiflows over such maps. In Section 4, we use operator renewal theory 
to reduce the nonuniformly expanding case to the Gibbs-Markov case. In Section 5 , we use an approximation argument to extend our results to the nonuniformly hyperbolic case.

\section{Statement of the Main Results}

In this section, we state our main results about rapid mixing. In Subsection 2.1, we consider the technically simpler case of nonuniformly expanding semiflows; here all definitions are given explicitly. In Subsection 2.2 we consider nonuniformly hyperbolic flows, referring to Young [35] for precise definitions.

2.1. Nonuniformly expanding semiflows. Let $(X, d)$ be a locally compact separable bounded metric space with Borel probability measure $m_{0}$ and let $T: X \rightarrow X$ be a nonsingular transformation for which $m_{0}$ is ergodic. Let $Y \subset X$ be a measurable subset with $m_{0}(Y)>0$, and let $\left\{Y_{j}\right\}$ be an at most countable measurable partition of $Y$ with $m_{0}\left(Y_{j}\right)>0$. We suppose that there is an $L^{1}$ return time function $r: Y \rightarrow \mathbb{Z}^{+}$, constant on each $Y_{j}$ with value $r(j) \geq 1$, and constants $\lambda>1$, $\eta \in(0,1), C \geq 1$ such that for each $j \geq 1$,

(1) $F=T^{r(j)}: Y_{j} \rightarrow Y$ is a bijection.

(2) $d(F x, F y) \geq \lambda d(x, y)$ for all $x, y \in Y_{j}$.

(3) $d\left(T^{\ell} x, T^{\ell} y\right) \leq C d(F x, F y)$ for all $x, y \in Y_{j}, 0 \leq \ell<r(j)$.

(4) $g_{j}=\frac{d\left(m_{0} \mid Y_{j} \circ F^{-1}\right)}{\left.d m_{0}\right|_{Y}}$ satisfies $\left|\log g_{j}(x)-\log g_{j}(y)\right| \leq C d(x, y)^{\eta}$ for all $x, y \in Y$.

Such a dynamical system $T: X \rightarrow X$ is called nonuniformly expanding. There is a unique $T$-invariant probability measure $m$ on $X$ equivalent to $m_{0}$ (see for example [36, Theorem 1]).

Remark 2.1. Discarding sets of zero measure, we have assumed without loss that the induced map $F: Y \rightarrow Y$ is defined everywhere on $Y$. This simplifies the formulation below of certain hypotheses involving periodic points.

Let $h: X \rightarrow \mathbb{R}^{+}$be a roof function such that for all $j \geq 1$,

(5) $h \in L^{\infty}(X)$ and $|h(x)-h(y)| \leq C d(x, y)^{\eta}$ for all $x, y \in T^{\ell} Y_{j}, 0 \leq \ell<r(j)$. Define the suspension $X^{h}=\{(x, u) \in X \times[0, \infty): u \in[0, h(x)]\} / \sim$ where $(x, h(x)) \sim(T x, 0)$. Define the suspension semiflow $T_{t}: X^{h} \rightarrow X^{h}$ by setting $T_{t}(x, u)=(x, u+t)$ computed modulo identifications. We obtain an invariant probability measure on $X^{h}$ given by $m^{h}=m \times \ell /|h|_{1}$ where $\ell$ denotes Lebesgue measure. For $m \geq 1, \eta>0$, let $C^{m, \eta}\left(X^{h}\right)$ consist of those $v: X^{h} \rightarrow \mathbb{R}$ for which $\|v\|_{m, \eta}=\|v\|_{\eta}+\left\|\partial_{t} v\right\|_{\eta}+\cdots+\left\|\partial_{t}^{m} v\right\|_{\eta}<\infty$, where $\partial_{t}$ denotes the derivative in the flow direction and

$$
\|v\|_{\eta}=|v|_{\infty}+\sup _{x \neq y}|v(x, u)-v(y, u)| / d(x, y)^{\eta} .
$$

Definition 2.2. The suspension semiflow $T_{t}$ is rapid mixing if for any $n \geq 1$ there exists $m \geq 1$ and $C \geq 1$ such that $\left|\rho_{v, w}(t)\right| \leq C\|v\|_{m, \eta}|w|_{\infty} t^{-n}$ for all $v \in C^{m, \eta}\left(X^{h}\right)$ and $w \in L^{\infty}\left(X^{h}\right)$, and all $t>0$.

Suppose that $Z \subset Y$ is a finite union of partition elements $Y_{j}$. Let $p \in Z$ be a periodic point for $F: Y \rightarrow Y$ such that $F^{i} p \in Z$ for all $i \geq 1$. We associate to $p$ the triple $(\tau, d, q) \in \mathbb{R}^{+} \times \mathbb{Z}^{+} \times \mathbb{Z}^{+}$where $\tau$ is the period of $p$ under the semiflow $T_{t}, d$ is the period under the map $T$, and $q$ is the period under the induced map $F$ (so $d=\sum_{i=0}^{q-1} r\left(F^{i} p\right)$ and $\tau=\sum_{i=0}^{d-1} h\left(T^{i} p\right)$ ). Let $\mathcal{T}_{Z}$ denote the set of such triples. 
Theorem 2.3. Let $T: X \rightarrow X$ be a nonuniformly expanding map and $h: X \rightarrow \mathbb{R}^{+}$ a roof function satisfying properties (1)-(5). Assume that $m_{0}(r>n)=O\left(\gamma^{n}\right)$ for some $\gamma \in(0,1)$. Let $Z \subset Y$ be a finite union of partition elements $Y_{j}$.

Suppose that the suspension semiflow $T_{t}: X^{h} \rightarrow X^{h}$ is not rapid mixing. Then there exist sequences $b_{k} \in \mathbb{R}$ with $\left|b_{k}\right| \rightarrow \infty$, and $\omega_{k}, \varphi_{k} \in[0,2 \pi)$, and constants $\alpha>0$ arbitrarily large, $C, \beta \geq 1$, such that

$$
\operatorname{dist}\left(b_{k} n_{k} \tau+\omega_{k} n_{k} d+q \varphi_{k}, 2 \pi \mathbb{Z}\right) \leq C q\left|b_{k}\right|^{-\alpha},
$$

for all $k \geq 1$ and all $(\tau, d, q) \in \mathcal{T}_{Z}$, where $n_{k}=\left[\beta \ln \left|b_{k}\right|\right]$.

Corollary 2.4. Let $T: X \rightarrow X$ be a nonuniformly expanding map and $h: X \rightarrow \mathbb{R}^{+}$ a roof function satisfying properties (1)-(5). Assume that $m_{0}(r>n)=O\left(\gamma^{n}\right)$ for some $\gamma \in(0,1)$. Fix four periodic solutions for $T_{t}: X^{h} \rightarrow X^{h}$ that each intersect $Y$, and let $\tau_{1}, \ldots, \tau_{4}$ be the periods. For Lebesgue almost all $\left(\tau_{1}, \cdots, \tau_{4}\right) \in\left(\mathbb{R}^{+}\right)^{4}$, the suspension semiflow $T_{t}: X^{h} \rightarrow X^{h}$ is rapid mixing.

Proof. Let $Z$ be the union of those partition elements $Y_{j}$ intersected by the four periodic solutions. We work with the triples $\left(\tau_{i}, d_{i}, q_{i}\right) \in \mathcal{T}_{Z}, i=1, \ldots, 4$, where $d_{i}, q_{i} \in \mathbb{Z}^{+}$. For simplicity, suppose that $q_{i}=1$.

Suppose that $T_{t}$ is not rapid mixing and let $\alpha>2$. Eliminating $\varphi_{k}$ from (2.1), we obtain $\operatorname{dist}\left(b_{k} n_{k} \tau_{12}+\omega_{k} n_{k} d_{12}, 2 \pi \mathbb{Z}\right) \leq 2 C\left|b_{k}\right|^{-\alpha}$ where $\tau_{12}=\tau_{1}-\tau_{2}$ and $d_{12}=$ $d_{1}-d_{2}$. Similar expressions hold for $\left(\tau_{13}, d_{13}\right)$ and $\left(\tau_{14}, d_{14}\right)$. Next, eliminate $\omega_{k}$ to obtain $\operatorname{dist}\left(b_{k} n_{k} \psi_{1}, 2 \pi \mathbb{Z}\right) \leq 2 C\left(d_{12}+d_{13}\right)\left|b_{k}\right|^{-\alpha}$ where $\psi_{1}=d_{13} \tau_{12}-d_{12} \tau_{13}$, and similarly for $\psi_{2}=d_{14} \tau_{12}-d_{12} \tau_{14}$.

Let $\alpha^{\prime} \in(2, \alpha)$. Arguing as in [18, Section 13], we obtain $\left(m_{1}, m_{2}\right) \in \mathbb{N}^{2}$ with $|m|=m_{1}+m_{2} \rightarrow \infty$ such that $m_{1} \psi_{1}+m_{2} \psi_{2}=O\left(|m|^{-\alpha^{\prime}}\right)$. This sequence of conditions is satisfied only by a measure zero set of pairs $\psi_{1}, \psi_{2}$. Hence for almost every $\left(\tau_{1}, \ldots, \tau_{4}\right)$ we obtain a contradiction, and so $T_{t}$ is rapid mixing.

Remark 2.5. Similarly, it suffices that there is a sequence of periodic orbits in $Z$ with good asymptotics in the sense of [19]. As shown in [19], good asymptotics is an open-dense condition for smooth systems. Hence, results on stable rapid mixing reduce to stability of the partition $\left\{Y_{j}\right\}$. We do not explore this issue further in this paper.

2.2. Nonuniformly hyperbolic flows. Let $(M, d)$ be a Riemannian manifold. Young [35] introduced a class of nonuniformly hyperbolic maps $T: M \rightarrow M$ (possibly with singularities) with the property that there is an ergodic $T$-invariant SRB measure for which exponential decay of correlations holds for Hölder observables. We refer to 35] for precise definitions, but some of the notions and notation are required to state our main results. (The further structure from [35] required for our proofs is postponed until Section 5.1.) In particular, there is a "uniformly hyperbolic" subset $Y \subset M$ with partition $\left\{Y_{j}\right\}$ and a return time function $r: Y \rightarrow \mathbb{Z}^{+}$ (denoted $R$ in [35]) constant on partition elements such that, modulo uniformly contracting directions, $F=T^{r(j)}: Y_{j} \rightarrow Y$ is a bijection.

The statement of our main result is completely analogous to that of Theorem 2.3 , Given a roof function $h: M \rightarrow \mathbb{R}^{+}$, the suspension flow $T_{t}: M^{h} \rightarrow M^{h}$ is defined as before. We define rapid mixing as in Definition 2.2 except that we now assume that both observables $v, w$ lie in $C^{m, \eta}\left(M^{h}\right)$ and $|w|_{\infty}$ is replaced by $\|w\|_{m, \eta}$. 
Suppose that $Z \subset Y$ is a finite union of partition elements $Y_{j}$. As in the nonuniformly expanding case, we define the set $\mathcal{T}_{Z}$ consisting of triples $(\tau, d, q)$ corresponding to periodic orbits for $F: Y \rightarrow Y$ lying entirely in $Z$.

Theorem 2.6. Let $T: M \rightarrow M$ be nonuniformly hyperbolic in the sense of Young 35] with $m_{0}(r>n)=O\left(\gamma^{n}\right)$ for some $\gamma \in(0,1)$. Let $h: M \rightarrow \mathbb{R}^{+}$be a roof function with $h \in L^{\infty}(M)$ and $|h(x)-h(y)| \leq C d(x, y)^{\eta}$ for all $x, y \in T^{\ell} Y_{j}$, $0 \leq \ell<r(j)$. Let $Z \subset Y$ be a finite union of partition elements $Y_{j}$.

If the suspension flow $T_{t}: M^{h} \rightarrow M^{h}$ is not rapid mixing, then condition (2.1) holds as in Theorem 2.3 .

Remark 2.7. Our criterion (2.1) for nonuniformly expanding/hyperbolic flows in Theorems 2.3 and 2.6 is more complicated than the corresponding criterion for uniformly hyperbolic flows [18, 19. In the uniformly hyperbolic case, the subset $Z$ and the sequence $\omega_{k}$ do not arise. Moreover, the phases $\varphi_{k}$ can be taken to be zero. A consequence is that a pair of periodic solutions suffices in Corollary 2.4.

It is not clear whether these extra complications can be removed with further work, or by using different techniques, but as shown by Corollary 2.4 and Remark 2.5. for many practical purposes the complications are not too serious.

\section{Suspensions of GibBs-Markov systems}

In this section, we consider rapid decay of correlations for a class of suspended Gibbs-Markov systems, where the roof function is piecewise Lipschitz (but not bounded).

We assume that $(Y, \mu)$ is a probability space, and that $\left\{Y_{j}, j \geq 1\right\}$ is a measurable partition of $Y$. Let $F: Y \rightarrow Y$ be a measure-preserving map. It is assumed that the partition $\left\{Y_{j}\right\}$ separates orbits of $F$ and that $\left.F\right|_{Y_{j}}: Y_{j} \rightarrow Y$ is a bijection for each $j$. If $a_{0}, \ldots, a_{n-1} \in\left\{Y_{j}\right\}$, we define the $n$-cylinder $\left[a_{0}, \ldots, a_{n-1}\right]=\bigcap_{i=0}^{n-1} F^{-i} a_{i}$. Fix $\theta \in(0,1)$ and define $d_{\theta}(x, y)=\theta^{s(x, y)}$ where the separation time $s(x, y)$ is the greatest integer $n \geq 0$ such that $x$ and $y$ lie in the same $n$-cylinder.

Define $F_{\theta}(Y)$ to be the Banach space of functions $v: Y \rightarrow \mathbb{R}$ that are Lipschitz with respect to $d_{\theta}$ with norm $\|v\|_{\theta}=|v|_{\infty}+|v|_{\theta}$ where $|v|_{\theta}$ is the least Lipschitz constant.

A function $v: Y \rightarrow \mathbb{R}$ is called piecewise Lipschitz if $\left.v\right|_{Y_{j}}$ is Lipschitz for each $j$. It is uniformly piecewise Lipschitz if the Lipschitz constants can be chosen independent of $j$. Note that $v \in F_{\theta}(Y)$ if and only if $v$ is uniformly piecewise Lipschitz and uniformly bounded.

We assume that $\mu$ is an invariant ergodic probability measure on $Y$. Define the potential function $p=\log \frac{d \mu}{d \mu \circ F}: Y \rightarrow \mathbb{R}$ and assume that $p$ is uniformly piecewise Lipschitz. In particular, $F: Y \rightarrow Y$ is Gibbs-Markov [1]. It follows in the usual way that there exists a constant $C_{1} \geq 1$ such that for all $x, y \in\left[a_{0}, \ldots, a_{k-1}\right]$,

$$
\left|\frac{e^{p_{k}(x)}}{e^{p_{k}(y)}}-1\right| \leq C_{1} \theta^{-k} d_{\theta}(x, y) \quad \text { and } \quad C_{1}^{-1} \leq \frac{\mu\left[a_{0}, \ldots, a_{k-1}\right]}{e^{p_{k}(x)}} \leq C_{1},
$$

where $p_{k}(x)=p(x)+p(F x)+\cdots+p\left(F^{k-1} x\right)$. (Note that in general $p \notin F_{\theta}(Y)$. Indeed, $p$ is bounded below if and only if the partition $\left\{Y_{j}\right\}$ is finite.)

Let $R: L^{1}(Y) \rightarrow L^{1}(Y)$ denote the transfer operator corresponding to $F: Y \rightarrow$ $Y$. So $\int_{Y} v w \circ F d \mu=\int_{Y} R v w d \mu$ for all $v \in L^{1}(Y)$ and $w \in L^{\infty}(Y)$. A calculation 
shows that $(R v)(x)=\sum_{F y=x} e^{p(y)} v(y)=\sum_{j \geq 1} e^{p\left(y_{j}\right)} v\left(y_{j}\right)$, where $y_{j}$ is the unique preimage of $x$ in $Y_{j}$.

Let $H: Y \rightarrow \mathbb{R}^{+}$be a piecewise Lipschitz roof function with $H \in L^{1}(Y)$.

Definition 3.1. The roof function $H$ has exponential tails if there is a partition $\left\{Z_{n}\right\}$ of $Y$ that is coarser than $\left\{Y_{j}\right\}$ such that $\mu\left(Z_{n}\right)=O\left(\gamma_{1}^{n}\right)$ and $\left\|1_{Z_{n}} H\right\|_{\theta}=O(n)$.

(We do not assume that $H$ is bounded nor that the Lipschitz constants of $1_{Y_{j}} H$ are bounded.)

Remark 3.2. Throughout this paper, $C_{1}, C_{2}, \ldots \geq 1$ denote universal constants that depend only on the Gibbs-Markov system $F: Y \rightarrow Y$, the partition $\left\{Y_{j}\right\}$, the metric $d_{\theta}$, the potential $p$ and the roof function $H$ (or the nonuniformly expanding map $T: X \rightarrow X$ and roof function $h: X \rightarrow \mathbb{R}$, etc., as appropriate). Similarly, $\gamma_{1}, \gamma_{2}, \ldots \in(0,1)$ denote universal constants.

Define the family of twisted transfer operators $R_{s}: L^{1}(Y) \rightarrow L^{1}(Y), s \in \mathbb{C}$,

$$
R_{s} v=R\left(e^{s H} v\right) \text {. }
$$

For purely imaginary $s=i b$, we define the one-sided inverses $M_{b}: L^{\infty}(Y) \rightarrow$ $L^{\infty}(Y)$,

$$
M_{b} v=e^{-i b H} v \circ F
$$

Definition 3.3. A subset $Z_{0} \subset Y$ is a finite subsystem of $Y$ if $Z_{0}=\bigcap_{n>1} F^{-n} Z$ where $Z$ is the union of finitely many elements from the partition $\left\{Y_{j}\right\}$. (Note that $\left.F\right|_{Z_{0}}: Z_{0} \rightarrow Z_{0}$ is a full one-sided shift on finitely many symbols.)

Definition 3.4. We say that $M_{b}$ has an approximate eigenfunction on a subset $Z \subset Y$ if there exist constants $\alpha>0$ arbitrarily large, $\beta>0$ and $C \geq 1$, and sequences $\left|b_{k}\right| \rightarrow \infty, \varphi_{k} \in[0,2 \pi), u_{k} \in F_{\theta}(Y)$ with $\left|u_{k}\right| \equiv 1$, such that setting $n_{k}=\left[\beta \ln \left|b_{k}\right|\right]$,

$$
\left|\left(M_{b_{k}}^{n_{k}} u_{k}\right)(y)-e^{i \varphi_{k}} u_{k}(y)\right| \leq C\left|b_{k}\right|^{-\alpha},
$$

for all $y \in Z$ and all $k \geq 1$.

Our main result in this section is the following result about the spectra of the twisted transfer operators $R_{s}$ for Gibbs-Markov maps.

Lemma 3.5. Let $F: Y \rightarrow Y$ be a Gibbs-Markov map and let $H: Y \rightarrow \mathbb{R}^{+}$be a piecewise Lipschitz roof function satisfying exponential tails. Let $Z_{0} \subset Y$ be a finite subsystem and suppose that $M_{b}$ has no approximate eigenfunctions on $Z_{0}$.

Then there exists $\alpha>0, \epsilon>0$ and $C \geq 1$ such that

$$
\left\|\left(I-R_{s}\right)^{-1}\right\|_{\theta} \leq C|b|^{\alpha} \text { for all } s=a+i b \text { with }|b|>1 \text { and }|a|<\epsilon|b|^{-\alpha} \text {. }
$$

Decay of correlations is a standard consequence of Lemma 3.5. For completeness, we state this result. Define the suspension semiflow $F_{t}: Y^{H} \rightarrow Y^{H}$ and define spaces of observables $F_{m, \theta}\left(Y^{H}\right)$ analogously to $C^{m, \eta}\left(X^{H}\right)$ as in Section 2.1. but with \|\|$_{\eta}$ replaced by \|\|$_{\theta}$.

Proposition 3.6. Let $F: Y \rightarrow Y$ be a Gibbs-Markov map and let $H: Y \rightarrow \mathbb{R}^{+}$ be a piecewise Lipschitz roof function satisfying exponential tails. Suppose further that the suspension flow $F_{t}: Y^{H} \rightarrow Y^{H}$ is mixing and that there exist constants $\epsilon>0$ and $C \geq 1$ such that $\left\|\left(I-R_{s}\right)^{-1}\right\|_{\theta} \leq C|b|^{\alpha}$ for all $s=a+i b$ with $|b|>1$ and $|a|<\epsilon|b|^{-\alpha}$. 
Then $F_{t}: Y^{H} \rightarrow Y^{H}$ is rapid mixing: for any $n \geq 1$, there exists $m \geq 1$ and $C \geq 1$ such that $\left|\rho_{v, w}(t)\right| \leq C\|v\|_{m, \theta}|w|_{\infty} t^{-n}$ for all $v \in F_{m, \theta}\left(Y^{H}\right), w \in L^{\infty}\left(Y^{H}\right)$, $t>0$.

Proof. Fix $k \geq 1$ and write $v=\tilde{v}+(v-\tilde{v})$ where $\tilde{v}$ is supported on the part of the suspension over $\bigcup_{j=1}^{k} Z_{j}$. Then $\rho_{v, w}(t)=\rho_{\tilde{v}, w}(t)+\rho_{v-\tilde{v}, w}(t)$ and $\left|\rho_{v-\tilde{v}, w}(t)\right| \leq$ $2|v|_{\infty}|w|_{\infty} \sum_{j>k} \mu\left(Z_{j}\right)\left|1_{Z_{j}} H\right|_{\infty} \leq C_{2}|v|_{\infty}|w|_{\infty} \gamma_{2}^{k}$.

The remaining term $\rho_{\tilde{v}, w}(t)$ is studied in the standard way $([17,28]$ and specifically [18, Section 10]) via the Laplace transform $\hat{\rho}_{\tilde{v}, w}(s)$. Ignoring an analytic term, $\hat{\rho}_{\tilde{v}, w}(s)=\int_{Y}\left[\left(I-R_{-s}\right)^{-1} v_{s}\right] w_{s} d \mu$ where $v_{s}(y)=\int_{0}^{H(y)} e^{s u} \tilde{v}(y, u) d u$ and $w_{s}(y)=\int_{0}^{H(y)} e^{-s u} w(y, u) d u$.

By exponential tails,

$$
\begin{aligned}
\left|w_{s}\right|_{1} & \leq \sum_{j \geq 1}\left|1_{Y_{j}} w_{s}\right|_{1} \leq \sum_{j \geq 1} \mu\left(Y_{j}\right)\left|1_{Y_{j}} w_{s}\right|_{\infty} \leq \sum_{j \geq 1} \mu\left(Y_{j}\right)\left|1_{Y_{j}} H\right|_{\infty} e^{\epsilon\left|1_{Y_{j}} H\right|_{\infty}}|w|_{\infty} \\
& \leq \sum_{n \geq 1} \mu\left(Z_{n}\right)\left|1_{Z_{n}} H\right|_{\infty} e^{\epsilon\left|1_{Z_{n}} H\right|_{\infty} \leq C_{3}|w|_{\infty},}
\end{aligned}
$$

and a straightforward calculation shows that $\left\|v_{s}\right\|_{\theta} \leq C_{4} k e^{\epsilon k}\|v\|_{\theta} \leq C_{5} e^{2 \epsilon k}\|v\|_{\theta}$. Hence $\left|\hat{\rho}_{\tilde{v}, w}(s)\right| \leq C^{\prime}|b|^{\alpha} e^{2 \epsilon k}\|v\|_{\theta}|w|_{\infty}$, for $|b|>1,|a|<\epsilon|b|^{-\alpha}$. Since $\left|\hat{\rho}_{v, w}(s)\right| \leq$ $|b|^{-m}\left|\hat{\rho}_{\partial_{t}^{m} v, w}(s)\right|$, we deduce that $\left|\hat{\rho}_{\tilde{v}, w}(s)\right| \leq C^{\prime}|b|^{\alpha-m} e^{2 \epsilon k}\|v\|_{m, \theta}|w|_{\infty}$, and it follows as in [18, Section 10] that for any $n \geq 1$, there exists $m \geq 1$ such that $\left|\rho_{\tilde{v}, w}(t)\right| \leq C^{\prime \prime} e^{2 \epsilon k} t^{-(n+1)}\|v\|_{m, \theta}|w|_{\infty}$ for $m$ sufficiently large. Hence

$$
\left|\rho_{v, w}(t)\right| \leq C^{\prime \prime}\left(e^{2 \epsilon k} t^{-(n+1)}+\gamma_{2}^{k}\right)\|v\|_{m, \theta}|w|_{\infty} .
$$

Taking $k=[(\ln t) /(2 \epsilon)]$ with $\epsilon$ sufficiently small yields the required result.

In the remainder of this section, we prove Lemma 3.5

3.1. Preliminary estimates. In this subsection, we write $s=a+i b$ and we carry out estimates for $0 \leq a<1$ and $b>1$. (The calculations are identical for $b<-1$, and simpler for $-1<a \leq 0$.)

Proposition 3.7. (a) $\left|R_{i b}\right|_{\infty} \leq 1$.

(b) $\left|R_{i b}^{n} v\right|_{\theta} \leq C_{6}\left\{b|v|_{\infty}+\theta^{n}|v|_{\theta}\right\}$ for all $n \geq 1$ and $v \in F_{\theta}(Y)$.

(c) $\left\|R^{n} v-\int_{Y} v\right\|_{\theta} \leq C_{7} \gamma_{3}^{n}\|v\|_{\theta}$ for all $n \geq 1$ and $v \in F_{\theta}(Y)$.

Proof. (a) is immediate and (c) follows from the quasicompactness [1, Section 4.7] of the transfer operator $R$. Part (b) is proved in Bruin et al. 10, where it is shown that $\left|R_{i b}^{n} v\right|_{\theta} \leq\left\{C_{1}^{2}+b C_{1} \theta(1-\theta)^{-1} \sum\left|1_{Y_{j}} H\right|_{\theta} \mu\left(Y_{j}\right)\right\}|v|_{\infty}+C_{1} \theta^{n}|v|_{\theta}$.

Remark 3.8. As in [18, Section 6], we define $\|v\|_{b}=\max \left\{|v|_{\infty},|v|_{\theta} /\left(2 C_{6} b\right)\right\}$. Then it follows from Proposition 3.7 that $\left\|R_{i b}^{n}\right\|_{b} \leq C_{6}+\frac{1}{2}$ for all $n \geq 1$. Moreover, $\left\|R_{i b}^{n}\right\|_{b} \leq 1$ for all $n \geq n_{0}\left(\right.$ where $\left.n_{0}=\left[\ln \left(2 C_{6}\right) /(-\ln \theta)\right]+1\right)$.

Proposition 3.9. For each $j \geq 1$, and all $v \in F_{\theta}(Y)$,

(a) $\left|R_{s} 1_{Y_{j}}\right|_{\infty} \leq C_{1} e^{a\left|1_{Y_{j}} H\right|_{\infty}} \mu\left(Y_{j}\right)$.

(b) $\left|R_{s} 1_{Y_{j}} v\right|_{\theta} \leq e^{a\left|1_{Y_{j}} H\right|_{\infty}} \mu\left(Y_{j}\right)\left\{\left(C_{1}^{2}+\theta C_{1}|s|\left|1_{Y_{j}} H\right|_{\theta}\right)|v|_{\infty}+\theta C_{1}|v|_{\theta}\right\}$.

(c) $\left\|R_{s} 1_{Y_{j}}\right\|_{b} \leq C_{8} e^{a\left|1_{Y_{j}} H\right|_{\infty}}\left(1+\left|1_{Y_{j}} H\right|_{\theta}\right) \mu\left(Y_{j}\right)$.

(d) $\left\|\left(R_{s}-R_{i b}\right) 1_{Y_{j}}\right\|_{b} \leq C_{9} a\left\|1_{Y_{j}} H\right\|_{\theta}\left(1+\left|1_{Y_{j}} H\right|_{\theta}\right) e^{a\left|1_{Y_{j}} H\right|_{\infty}} \mu\left(Y_{j}\right)$. 
Proof. For $x \in Y$, we have $\left(R 1_{Y_{j}} v\right)(x)=e^{p(y)} v(y)$ where $y$ is the unique preimage of $x$ in $Y_{j}$. Hence $\left|R 1_{Y_{j}}\right|_{\infty} \leq e^{\left|1_{Y_{j}} p\right|_{\infty}} \leq C_{1} \mu\left(Y_{j}\right)$. Moreover,

$$
\begin{aligned}
\left|\left(R 1_{Y_{j}} v\right)(x)-\left(R 1_{Y_{j}} v\right)\left(x^{\prime}\right)\right| & \leq\left|e^{p(y)}-e^{p\left(y^{\prime}\right)}\right||v(y)|+e^{p\left(y^{\prime}\right)}\left|v(y)-v\left(y^{\prime}\right)\right| \\
& \leq e^{p\left(y^{\prime}\right)}\left|e^{p(y)-p\left(y^{\prime}\right)}-1\right||v|_{\infty}+e^{p\left(y^{\prime}\right)} \theta|v|_{\theta} d_{\theta}\left(x, x^{\prime}\right) \\
& \leq C_{1} \mu\left(Y_{j}\right)\left\{C_{1} d_{\theta}\left(x, x^{\prime}\right)|v|_{\infty}+\theta|v|_{\theta} d_{\theta}\left(x, x^{\prime}\right)\right\}
\end{aligned}
$$

so that $\left|R 1_{Y_{j}} v\right|_{\theta} \leq \mu\left(Y_{j}\right)\left\{C_{1}^{2}|v|_{\infty}+\theta C_{1}|v|_{\theta}\right\}$.

Next, write $R_{s} 1_{Y_{j}} v=R 1_{Y_{j}}\left(e^{s 1_{Y_{j}} H} v\right)$. Using the inequality

$$
\left|e^{z}-e^{w}\right| \leq \sqrt{2}|z-w| e^{\max \{\Re z, \Re w\}} \quad \text { for } z, w \in \mathbb{C},
$$

we obtain $\left|e^{s 1_{Y_{j}} H}\right|_{\theta} \leq \sqrt{2}|s|\left|1_{Y_{j}} H\right|_{\theta} e^{|\Re s|\left|1_{Y_{j}} H\right|_{\infty}}$. Parts (a) and (b) follow easily. Part (c) follows from parts (a) and (b) and the definition of \|\|$_{b}$.

To prove part (d), we write $\left(R_{s}-R_{i b}\right) 1_{Y_{j}}=R_{i b} 1_{Y_{j}}\left(e^{a 1_{Y_{j}} H}-1\right)$. It suffices to estimate $e^{a 1_{Y_{j}} H}-1$ and substitute into the estimates for $R_{i b} 1_{Y_{j}}$. Since $a \geq 0$, $\left|e^{a 1_{Y_{j}} H(x)}-1\right|=e^{a 1_{Y_{j}} H(x)}-1 \leq e^{a\left|1_{Y_{j}} H\right|_{\infty}}-1$. Also, $\left|e^{a 1_{Y_{j}} H}-1\right|_{\theta}=\left|e^{a 1_{Y_{j}} H}\right|_{\theta} \leq$ $e^{a\left|1_{Y_{j}} H\right|_{\infty}} a\left|1_{Y_{j}} H\right|_{\theta}$. The result follows.

3.2. Proof of Lemma 3.5. Consider the following conditions:

(A) There exists a finite subsystem $Z_{0} \subset Y$ such that $M_{b}$ has no approximate eigenfunctions on $Z_{0}$.

(B) There exists $\alpha>0$ such that $\left\|\left(I-R_{i b}\right)^{-1}\right\|_{b}=O\left(|b|^{\alpha}\right)$ as $|b| \rightarrow \infty$.

(C) There exists $\alpha, \epsilon>0, C \geq 1$ such that $\left\|\left(I-R_{s}\right)^{-1}\right\|_{b} \leq C|b|^{\alpha}$ for all $s=a+i b$ with $|b|>1$ and $|a|<\epsilon|b|^{-\alpha}$.

Adapting arguments of Dolgopyat [18, we show that (A) implies (B), and that (B) implies (C).

\section{Condition (B) implies condition (C).}

Proposition 3.10. There exist $\epsilon>0$ such that $\left\|R_{s}-R_{i b}\right\|_{b} \leq C_{10}|a|$, for all $s=a+i b$ with $|b|>1$ and $0 \leq|a|<\epsilon$.

Proof. Write $R_{s}-R_{i b}=\sum_{j \geq 1}\left(R_{s}-R_{i b}\right) 1_{Y_{j}}$. We estimated $\left\|\left(R_{s}-R_{i b}\right) 1_{Y_{j}}\right\|_{b}$ in Proposition 3.9(d). By exponential tails, we have that $\left\|R_{s}-R_{i b}\right\|_{b}$ is dominated by a uniformly convergent series for $|a| \leq \epsilon$. Moreover, the series vanishes at $a=0$ and is $C^{1}$ on $[-\epsilon, \epsilon]$. It follows that $\left\|R_{s}-R_{i b}\right\|_{b}=O(a)$ on $[-\epsilon, \epsilon]$ uniformly in $b$.

Corollary 3.11. Condition (B) implies condition (C).

Proof. Following [18, Section 2], write $\left(I-R_{s}\right)^{-1}=\left(I-R_{i b}\right)^{-1}(I-A)^{-1}$, where $A=\left(I-R_{i b}\right)^{-1}\left(R_{s}-R_{i b}\right)$. By condition (B) and Proposition 3.10 there exist constants $C \geq 1, \epsilon_{1}>0$ such that $\|A\|_{b} \leq C|a \| b|^{\alpha}$ for all $|a|<\epsilon_{1}$ and $|b|>1$. Hence there exists $\epsilon>0$ such that $\|A\|_{b}<\frac{1}{2}$ for $|a|<\epsilon|b|^{-\alpha}$. Therefore $\left\|(I-A)^{-1}\right\|_{b} \leq 2$, and the result follows. 
Condition (A) implies condition (B). In this part of the proof, we restrict our attention to $s=i b$ where $b>1$ (the results are identical for $|b|>1$ ).

Since we are estimating operator norms with respect to \|\|$_{b}$, we consider the unit ball $F_{\theta}(Y)_{b}=\left\{v \in F_{\theta}:\|v\|_{b} \leq 1\right\}$. It follows from Remark 3.8 that $\left|R_{i b}^{n} v\right|_{\infty} \leq 1$ and $\left|R_{i b}^{n} v\right|_{\theta} \leq 2 C_{6} b$ for all $v \in F_{\theta}(Y)_{b}$ and $n \geq n_{0}$.

Throughout, $Z$ denotes a fixed subset of $Y$ consisting of a finite union of partition elements of $Y$, and $Z_{0}=\bigcap_{j \geq 0} F^{-j} Z$. Note that $p$ is uniformly bounded on $Z_{0}$ and moreover $\left|p_{n}(x)\right| \leq n\left|1_{Z_{0}} p\right|_{\infty}$ for all $x \in Z_{0}$ and $n \geq 1$.

Lemma 3.12. Fix $\alpha_{2}>0$. Then there exist $\alpha_{1}>0$ and $\beta>0$, such that the following is true for each fixed $b>2$, setting $n(b)=[\beta \ln b]$ :

Suppose that there exists $v_{0} \in F_{\theta}(Y)_{b}$ such that for all $x \in Z_{0}$ and all $j=0,1,2$,

$$
\left|\left(R_{i b}^{j n(b)} v_{0}\right)(x)\right| \geq 1-1 / b^{\alpha_{1}} .
$$

Then there exists $w \in F_{\theta}(Y),|w(x)| \equiv 1$, and $\varphi \in[0,2 \pi)$ such that for all $y \in Z_{0}$,

$$
\left|\left(M_{b}^{n(b)} w\right)(y)-e^{i \varphi} w(y)\right| \leq 8 / b^{\alpha_{2}} .
$$

Proof. We write $n=n(b)$ and $C_{11}=16 C_{6}$. Set

$$
\beta=\left(\alpha_{2}+2+\ln C_{11} / \ln 2\right) /(-\ln \theta), \quad \alpha_{1}=\max \left\{1,2 \alpha_{2}+\beta\left|1_{Z_{0}} p\right|_{\infty}\right\} .
$$

Following [18, Section 8], we write $v_{j}=R_{i b}^{j n} v_{0}$ and $v_{j}=s_{j} w_{j}$, where $\left|w_{j}(x)\right| \equiv 1$ and $1-1 / b^{\alpha_{1}} \leq s_{j}(x) \leq 1$ for $x \in Z_{0}$. Note that $\left|v_{j}\right|_{\theta} \leq 2 C_{6} b$ so that $\left|w_{j}\right|_{\theta} \leq C_{11} b$. Rearrange $v_{1}=R_{i b}^{n} v_{0}$ to obtain $w_{1}^{-1} R_{i b}^{n}\left(s_{0} w_{0}\right)=s_{1} \geq 1-1 / b^{\alpha_{1}}$. It then follows from the definition of $R_{i b}$ that $e^{p_{n}(y)}\left[1-\Re\left(e^{i b H_{n}(y)} w_{0}(y) w_{1}^{-1}\left(F^{n} y\right)\right)\right] \leq 1 / b^{\alpha_{1}}$ for all $y \in Y$ with $F^{n} y \in Z_{0}$. Hence $\left|e^{i b H_{n}(y)} w_{0}(y)-w_{1}\left(F^{n} y\right)\right| \leq 2\left(e^{-p_{n}(y)} / b^{\alpha_{1}}\right)^{1 / 2}$. Similarly, with $w_{0}$ and $w_{1}$ replaced by $w_{1}$ and $w_{2}$. Restricting to $y \in Z_{0}$, we have $e^{-p_{n}(y)} / b^{\alpha_{1}} \leq 1 / b^{2 \alpha_{2}}$ and hence

$$
\left|e^{i b H_{n}(y)} w_{0}(y)-w_{1}\left(F^{n} y\right)\right| \leq 2 / b^{\alpha_{2}}, \quad\left|e^{i b H_{n}(y)} w_{1}(y)-w_{2}\left(F^{n} y\right)\right| \leq 2 / b^{\alpha_{2}},
$$

for all $y \in Z_{0}$. Fix $z \in Z_{0}$ and define $w_{j}(z)=e^{i \varphi_{j}}$ for $j=0,1$. To each $y$, we associate $y^{*}=z_{0} \cdots z_{n-1} y_{n} y_{n+1} \cdots \in Z_{0}$. Then $y^{*}$ is within distance $\theta^{n}$ of $z$ and $F^{n} y^{*}=F^{n} y$. We obtain

$$
\begin{aligned}
& \left|e^{i b H_{n}\left(y^{*}\right)} e^{i \varphi_{0}}-w_{1}\left(F^{n} y\right)\right| \leq 2 / b^{\alpha_{2}}+C_{11} b \theta^{n} \leq 3 / b^{\alpha_{2}} \\
& \left|e^{i b H_{n}\left(y^{*}\right)} e^{i \varphi_{1}}-w_{2}\left(F^{n} y\right)\right| \leq 2 / b^{\alpha_{2}}+C_{11} b \theta^{n} \leq 3 / b^{\alpha_{2}}
\end{aligned}
$$

(by the choice of $\beta$ ), and so $\left|e^{-i \varphi} w_{1}\left(F^{n} y\right)-w_{2}\left(F^{n} y\right)\right| \leq 6 / b^{\alpha_{2}}$. Substituting into (3.2) yields the required approximate eigenfunction $w=w_{1}$.

Lemma 3.13. For any $\alpha_{1}, \beta>0$, there exists $\alpha>0$ and $C \geq 1$ with the following property.

Let $b \geq 1$ and suppose that for any $v \in F_{\theta}(Y)_{b}$ there exists $x_{0} \in Z_{0}$ and $j \leq$ $[\beta \ln b]$ such that $\left|R_{i b}^{j} v\left(x_{0}\right)\right| \leq 1-1 / b^{\alpha_{1}}$. Then $\left\|\left(I-R_{i b}\right)^{-1}\right\|_{b} \leq C b^{\alpha}$.

Proof. Following [18, Section 7], we use the pointwise estimate on iterates of $R_{i b}$ to obtain estimates on the $L^{1}, L^{\infty}$ and \|\|$_{b}$ norms.

Write $\hat{u}=R_{i b}^{j} v$ and $u=R_{i b}^{\ell(b)} v$ where $\ell(b)=[\beta \ln b]$. Note that $|\hat{u}|_{\infty} \leq 1$ and $|\hat{u}|_{\theta} \leq 2 C_{6} b$. Hence, $|\hat{u}(x)| \leq 1-1 /\left(2 b^{\alpha_{1}}\right)$ for all $x$ within distance $1 /\left(4 C_{6} b^{\alpha_{1}+1}\right)$ of $x_{0}$. Call this subset $U$. If $\mathcal{C}_{k}$ is a $k$-cylinder, then $\operatorname{diam} \mathcal{C}_{k}=\theta^{k}$, so provided 
$\theta^{k}<1 /\left(4 C_{6} b^{\alpha_{1}+1}\right)$, the $k$-cylinder containing $x_{0}$ lies inside $U$. It suffices to take $k \sim\left(\alpha_{1}+1\right) \ln b /(-\ln \theta)$. By (3.1),

$$
\mu(U) \geq \mu\left(\mathcal{C}_{k}\right) \geq C_{1}^{-1} e^{-p_{k}\left(x_{0}\right)} \geq C_{1}^{-1} e^{-k\left|1_{Z_{0}} p\right|_{\infty}} \geq C_{12}^{-1} b^{-\left(\alpha_{1}+1\right) \alpha_{2}},
$$

where $\alpha_{2}=\left|1_{Z_{0}} p\right|_{\infty} /(-\ln \theta)$. Breaking up $Y$ into $U$ and $Y-U$,

$$
|u|_{1} \leq|\hat{u}|_{1} \leq\left(1-1 /\left(2 b^{\alpha_{1}}\right)\right) \mu(U)+1-\mu(U)=1-\mu(U) /\left(2 b^{\alpha_{1}}\right) \leq 1-C_{13}^{-1} b^{-\alpha_{3}},
$$

where $\alpha_{3}=\alpha_{1}+\alpha_{2}+\alpha_{1} \alpha_{2}$. Now,

$$
\begin{aligned}
\left|R_{i b}^{n} u\right|_{\infty} & \leq\left|\left(R^{n}|u|\right)\right|_{\infty} \leq\left|\left(R^{n}|u|-\int|u|\right)\right|_{\infty}+|u|_{1} \leq C_{7} \gamma_{3}^{n}\|u\|_{\theta}+|u|_{1} \\
& \leq\left(1+2 C_{6} b\right) C_{7} \gamma_{3}^{n}+1-C_{13}^{-1} b^{-\alpha_{3}} .
\end{aligned}
$$

Choosing $n=n_{1}(b)=\left[\beta_{1} \ln b\right]$ where $\beta_{1} \gg 1$ ensures that

$$
\left|R_{i b}^{\ell(b)+n_{1}(b)} v\right|_{\infty}=\left|R_{i b}^{n_{1}(b)} u\right|_{\infty} \leq 1-C_{14}^{-1} b^{-\alpha_{3}} .
$$

Setting $n_{2}(b)=\left[\beta_{2} \ln b\right]$ where $\beta_{2}=\beta+\beta_{1}$,

$$
\left|R_{i b}^{n_{2}(b)} v\right|_{\infty} \leq 1-C_{14}^{-1} b^{-\alpha_{3}} .
$$

By Proposition 3.7 (a), (b), $\left|R_{i b}^{n_{2}(b)+n}\right|_{\infty} \leq 1-C_{14}^{-1} b^{-\alpha_{3}}$ for all $n \geq 0$, and

$$
\left|R_{i b}^{n_{2}(b)+n} v\right|_{\theta} /\left(2 C_{6} b\right) \leq \frac{1}{2}+\theta^{n} C_{6} \leq \frac{3}{4},
$$

for $n$ sufficiently large (independent of $b$ ). Increasing $\beta_{2}$ slightly, $\left\|R_{i b}^{n_{2}(b)} v\right\|_{b} \leq$ $1-C_{14}^{-1} b^{-\alpha_{3}}$. Hence $\left\|\left(I-R_{i b}^{n_{2}(b)}\right)^{-1}\right\|_{b} \leq C_{14} b^{\alpha_{3}}$. Using the identity $(I-A)^{-1}=$ $\left(I+A+\cdots+A^{m-1}\right)\left(I-A^{m}\right)^{-1}$ and Remark 3.8, we obtain

$$
\left\|\left(I-R_{i b}\right)^{-1}\right\|_{b}=O\left(n_{2}(b) b^{\alpha_{3}}\right)=O\left(b^{\alpha}\right),
$$

for any choice of $\alpha>\alpha_{3}$.

Combining Lemmas 3.12 and 3.13 , we obtain that condition (A) implies condition (B). This completes the proof of Lemma 3.5.

3.3. A generalisation of Lemma 3.5. We continue to suppose that $F: Y \rightarrow Y$ is Gibbs-Markov and that $H: Y \rightarrow \mathbb{R}^{+}$is a piecewise Lipschitz roof function. Suppose that $r: Y \rightarrow \mathbb{Z}^{+}$is constant on partition elements with value $r(j)$ on $Y_{j}$. By exponential tails, we mean that there is a coarser partition $\left\{Z_{n}\right\}$ with $\mu\left(Z_{n}\right)=O\left(\gamma_{1}^{n}\right),\left\|1_{Z_{n}} H\right\|_{\theta}=O(n)$ and $\left|1_{Z_{n}} r\right|_{\infty}=O(n)$.

For $z \in \mathbb{C}$, define

$$
R_{s, z} v=R_{s}\left(e^{z r} v\right)=R\left(e^{s H} e^{z r} v\right) .
$$

We continue to write $z=a+i b$ restricting to $|b|>1$, and we write $z=\sigma+i \omega$ with the natural restriction $\omega \in[0,2 \pi)$ (since $r$ is integer valued). Define

$$
M_{b, \omega} v=M_{b}\left(e^{-i r \omega} v\right)=e^{-i b H} e^{-i \omega r} v \circ F .
$$

We say that $M_{b, \omega}$ has an approximate eigenfunction on a subset $Z \subset Y$ if there exist constants $\alpha>0$ arbitrarily large, $\beta>0$ and $C \geq 1$, and sequences $\left|b_{k}\right| \rightarrow \infty, \omega_{k} \in$ $[0,2 \pi), \varphi_{k} \in[0,2 \pi), u_{k} \in F_{\theta}(Y)$ with $\left|u_{k}\right| \equiv 1$, such that setting $n_{k}=\left[\beta \ln \left|b_{k}\right|\right]$,

$$
\left|\left(M_{b_{k}, \omega_{k}}^{n_{k}} u_{k}\right)(y)-e^{i \varphi_{k}} u_{k}(y)\right| \leq C\left|b_{k}\right|^{-\alpha}
$$

for all $y \in Z$ and all $k \geq 1$. 
Lemma 3.14. Let $F: Y \rightarrow Y$ be a Gibbs-Markov map, let $H: Y \rightarrow \mathbb{R}^{+}$be a piecewise Lipschitz roof function and let $r: Y \rightarrow \mathbb{Z}^{+}$be constant on partition elements. Assume exponential tails. Let $Z_{0} \subset Y$ be a finite subsystem and suppose that $M_{b, \omega}$ has no approximate eigenfunctions on $Z_{0}$.

Then there exists $\alpha>0, \epsilon>0$ and $C \geq 1$ such that

$$
\left\|\left(I-R_{s, z}\right)^{-1}\right\|_{b} \leq C|b|^{\alpha} \quad \text { for all }|b|>1, \omega \in[0,2 \pi),|a|,|\sigma|<\epsilon|b|^{-\alpha} .
$$

Proof. This is identical to the proof of the corresponding statements with $z=0$. Note that the $e^{z r}$ factor is analogous to the $e^{s H}$ term but is easier to handle (since $r$ is integer-valued and constant on partition elements).

\section{RAPID MIXING FOR NONUNIFORMLY EXPANDING SEMIFLOWS}

In this section we prove Theorem 2.3 . We begin by considering suspension semiflows over nonuniformly expanding Young towers 36. These towers are Markov systems (even though the underlying map need not be Markov) with additional distortion properties. Essentially, a Markov system $\Delta$ over a base $Y$ is a tower if the induced map on the base is Gibbs-Markov.

In Subsection 4.1, we introduce the tower maps $f: \Delta \rightarrow \Delta$ and roof functions to be studied in this section. In Subsection 4.2. we use operator renewal theory to reduce the tower case down to the Gibbs-Markov case $F: Y \rightarrow Y$ studied in Section 3 . In Subsection 4.3 , we prove Theorem 2.3 by modelling the nonuniformly expanding map $T: X \rightarrow X$ by a tower map $f: \Delta \rightarrow \Delta$.

4.1. Return times and towers. Let $F: Y \rightarrow Y$ be a Gibbs-Markov map with invariant measure $\mu$, partition $\left\{Y_{j}\right\}$, separation time $s(x, y)$ and metric $d_{\theta}(x, y)$ as in Section 3. Consider a return time function $r: Y \rightarrow \mathbb{Z}^{+}$that is constant on partition elements. Assume that $r \in L^{1}$ and let $r(j)$ be the value of $r$ on $Y_{j}$. We form a tower $\Delta$ with base $Y$ as a discrete suspension, so $\Delta=\{(y, \ell) \in Y \times \mathbb{N}: \ell \leq r(y)\} / \sim$ where $(y, r(y)) \sim(F y, 0)$. Define the tower map $f: \Delta \rightarrow \Delta$ by setting $f(y, \ell)=(y, \ell+1)$ computed modulo identifications. We obtain an invariant probability measure (also denoted by $\mu$ ) on $\Delta$ given by $\mu \times \nu /|r|_{1}$ where $\nu$ denotes counting measure. Also we have a countable partition on $\Delta$ given by $\left\{\Delta_{j, \ell}: 0 \leq \ell<r(j)\right\}$ where $\Delta_{j, \ell}=$ $Y_{j} \times\{\ell\}$. The separation time $s: Y \times Y \rightarrow \mathbb{N}$ in Section 3 extends to the tower as follows. If $x$ and $y$ lie in distinct partition elements, then $s(x, y)=0$. If $x, y \in \Delta_{j, \ell}$, then there exist unique $x^{\prime}, y^{\prime} \in \Delta_{j, 0}$ such that $x=f^{\ell} x^{\prime}$ and $y=f^{\ell} y^{\prime}$. Regarding $x^{\prime}, y^{\prime}$ as elements of $Y_{j}$, set $s(x, y)=s\left(x^{\prime}, y^{\prime}\right)$. This defines the separation time $s: \Delta \times \Delta \rightarrow \mathbb{N}$ and hence a metric $d_{\theta}(x, y)=\theta^{s(x, y)}$ on $\Delta$. Let $F_{\theta}(\Delta)$ denote the Banach space of Lipschitz functions $v: \Delta \rightarrow \mathbb{R}$ with norm $\|v\|_{\theta}=|v|_{\infty}+|v|_{\theta}$.

We recover the Gibbs-Markov map $F: Y \rightarrow Y$ as the induced map $F(y)=$ $f^{r(y)}(y)$.

Let $h: \Delta \rightarrow \mathbb{R}^{+}$be a Lipschitz roof function $\left(h \in F_{\theta}(\Delta)\right)$, and define the induced roof function $H: Y \rightarrow \mathbb{R}^{+}$given by $H(y)=h_{r(y)}(y)=\sum_{j=0}^{r(y)-1} h \circ f^{j}(y)$.

Define the partition $\left\{Z_{n}\right\}$ of $Y$ where $Z_{n}=\{y \in Y: r(y)=n\}$. This partition is coarser than $\left\{Y_{n}\right\}$ and $1_{Z_{n}} r=n$ by definition. Moreover, a calculation using the definition of $d_{\theta}$ on $\Delta$ shows that $\left\|1_{Z_{n}} H\right\|_{\theta} \leq n\|h\|_{\theta}$. Hence, $F, H$ and $r$ satisfy the exponential tails condition in Section 3.3 if and only if $\mu\left(Z_{n}\right)=O\left(\gamma_{1}^{n}\right)$. In this case, we say that the tower $\Delta$ has exponential tails. 
Proposition 4.1. If $\Delta$ has exponential tails, then there exists $\epsilon_{1}>0$ such that $\left\|R_{s} 1_{Z_{n}}\right\|_{b} \leq C_{15} \gamma_{4}^{n}$ for all $n \geq 1$ and all $s=a+i b$ with $0 \leq a<\epsilon_{1}$ and $b>1$.

Proof. Applying Proposition [3.9(c),

$$
\begin{aligned}
\left\|R_{s} 1_{Z_{n}}\right\|_{b} & =\sum_{j: r(j)=n}\left\|R_{s} 1_{Y_{j}}\right\|_{b} \leq C_{8} \sum_{j: r(j)=n} e^{a\left|1_{Y_{j}} H\right|_{\infty}}\left(1+\left|1_{Y_{j}} H\right|_{\theta}\right) \mu\left(Y_{j}\right) \\
& \leq C_{8} \mu\left(Z_{n}\right) e^{a n|h|_{\infty}\left(1+n|h|_{\theta}\right) .}
\end{aligned}
$$

Now choose $\epsilon_{1}>0$ and $\gamma_{4}>0$ so that $\gamma_{4}<\gamma_{1} e^{\epsilon_{1}\|h\|_{\theta}}<1$.

4.2. Renewal theory. Let $L: L^{1}(\Delta) \rightarrow L^{1}(\Delta)$ denote the transfer operator corresponding to $f: \Delta \rightarrow \Delta$. Let $L_{s} v=L\left(e^{s h} v\right)$ be the twisted transfer operator for $s \in \mathbb{C}$. Then $\left(L_{s}^{n} v\right)(x)=\sum_{f^{n} z=x} g_{n}(z) e^{s h_{n}(z)} v(z)$ where $g_{n}(z)$ is the inverse of the Jacobian of $f^{n}$ at $z$.

Renewal theory gives a mechanism for relating estimates of $L_{s}^{n}, n \geq 1$, to estimates of $R_{s}$ (where $R_{s}$ is the twisted transfer operator introduced in Section 3 corresponding to the Gibbs-Markov system $F: Y \rightarrow Y$ ). Following Sarig [31] (see also [20, 10]) we define $T_{s, n}: L^{1}(Y) \rightarrow L^{1}(Y)$ and $R_{s, n}: L^{1}(Y) \rightarrow L^{1}(Y)$ by

$$
T_{s, n} v=1_{Y} L_{s}^{n}\left(1_{Y} v\right), \quad R_{s, n} v=1_{Y} L_{s}^{n}\left(1_{Z_{n}} v\right) .
$$

We have the identifications $R_{s, n}=R_{s} 1_{Z_{n}}$ and $R_{s}=\sum_{n \geq 1} R_{s, n}$. For $z \in \mathbb{C}$, define

$$
T_{s, z}=I+\sum_{n \geq 1} T_{s, n} e^{z n}, \quad R_{s, z}=\sum_{n \geq 1} R_{s, n} e^{z n}
$$

Proposition 4.2 (Renewal equation). Let $s \in \mathbb{C}$. Assume that $\omega \mapsto R_{s, i \omega}$ is $C^{1}$ and that $I-R_{s, i \omega}$ is invertible for $\omega \in[0,2 \pi)$. Then $T_{s, i \omega}=\left(I-R_{s, i \omega}\right)^{-1}$.

Proof. Define $\hat{T}_{s, i \omega}=\left(I-R_{s, i \omega}\right)^{-1}$. Then $\omega \mapsto \hat{T}_{s, i \omega}$ is $C^{1}$ and hence has a convergent Fourier series with coefficients $\hat{T}_{s, n}$ satisfying $\hat{T}_{s, n}=\sum_{k=1}^{n} \hat{T}_{s, n-k} R_{s, k}$ and $\hat{T}_{0}=I$.

We claim that $T_{s, n}=\sum_{k=1}^{n} T_{s, n-k} R_{s, k}$ (with $T_{0}=I$ ). It then follows that $T_{s}=\hat{T}_{s}=\left(I-R_{s}\right)^{-1}$ as required. To prove the claim, compute that

$$
\begin{aligned}
\int_{\Delta}\left(T_{s, n-k} R_{s, k} v\right) w & =\int_{\Delta}\left(L_{s}^{n-k} 1_{Y} L_{s}^{k} 1_{Z_{k}} v\right)\left(1_{Y} w\right) \\
& =\int_{\Delta}\left(e^{s h_{n-k}} 1_{Y} L_{s}^{k} 1_{Z_{k}} v\right)\left(1_{Y} w\right) \circ f^{n-k} \\
& =\int_{\Delta}\left(e^{s h_{k}} 1_{Z_{k}} v\right)\left(1_{Y} w\right) \circ f^{n} e^{s h_{n-k} \circ f^{k}} 1_{Y} \circ f^{k} \\
& =\int_{\Delta}\left(e^{s h_{n}} v\right)\left(w \circ f^{n}\right) 1_{Z_{k}} 1_{Y} \circ f^{n} .
\end{aligned}
$$

On the other hand, $\int_{\Delta}\left(T_{s, n} v\right) w=\int_{\Delta} e^{s h_{n}} v\left(w \circ f^{n}\right) 1_{Y} 1_{Y} \circ f^{n}$. The result follows since $\bigcup_{k=1}^{n} Z_{k} \cap f^{-n} Y=Y \cap f^{-n} Y$.

It should be noted that $R_{s, z} v=R\left(e^{s H} e^{z r} v\right)$ coincides with the operator defined in Section 3.3. (Recall that the return time function $r$ takes the value $n$ on $Z_{n}$.) Lemma 3.14 gives conditions under which $\left\|\left(I-R_{s, z}\right)\right\|_{b}^{-1} \leq C|b|^{\alpha}$ holds. 
Lemma 4.3. Suppose that there exist constants $\alpha, \epsilon>0, C \geq 1$ such that

$$
\left\|\left(I-R_{s, z}\right)^{-1}\right\|_{b} \leq C|b|^{\alpha},
$$

for all $s=a+i b, z=\sigma+i \omega$ with $|b|>1, \omega \in[0,2 \pi)$ and $|a|,|\sigma|<\epsilon|b|^{-\alpha}$.

Then there exists $\delta>0$ such that

$$
\left\|T_{s, n}\right\|_{b} \leq C|b|^{\alpha} e^{-n \delta|b|^{-\alpha}}
$$

for all $n \geq 1$, and $s=a+i b$ with $|a|<\epsilon|b|^{-\alpha}$ and $|b|>1$.

Proof. Write $R_{s, z}=\sum_{n \geq 1} R_{s} 1_{Z_{n}} e^{z n}$.

Restricting to $z=i \omega$, this is a Fourier series with exponentially decaying Fourier coefficients by Proposition 4.1. Hence, the series continues analytically to an annulus $e^{\sigma+i \omega},|\sigma|<\delta_{0}$ for some $\delta_{0}>0$. By the renewal equation, for each such $s$, the Fourier series $T_{s, i \omega}=\left(I-R_{s, i \omega}\right)^{-1}$ has an analytic extension to the annulus $e^{\sigma+i \omega}$ with $|\sigma|<\epsilon|b|^{-\alpha}$. Hence, the Fourier coefficients $T_{s, n}$ decay at the required rate for any $\delta<\epsilon$.

Lemma 4.4. Assume the hypotheses of Lemma 4.3. Then there exist constants $\alpha, \delta, \epsilon>0, C \geq 1$, such that

$$
\left|L_{s}^{n} v\right|_{1} \leq C|b|^{\alpha} e^{-n \delta|b|^{-\alpha}}\|v\|_{b},
$$

for all $v \in F_{\theta}(\Delta), n \geq 1$, and $s=a+i b$ with $|b|>1$ and $|a|<\epsilon|b|^{-\alpha}$.

Proof. Recall that $\left(L_{s}^{n} v\right)(x)=\sum_{f^{n} z=x} g_{n}(z) e^{s h_{n}(z)} v(z)$. Following Gouëzel 22] (see also [21, 10]) we write $L_{s}^{n}=\sum_{i+j+k=n} A_{s, i} T_{s, j} B_{s, k}+E_{s, n}$, where

$$
\begin{aligned}
& \left(T_{s, n} v\right)(x)=\sum_{\substack{f^{n} z=x \\
x, z \in Y}}, \quad\left(A_{s, n} v\right)(x)=\sum_{\substack{f^{n} z=x \\
z \in Y ; f z \notin Y, \ldots, f^{n} z \notin Y}}, \\
& \left(E_{s, n} v\right)(x)=\sum_{\substack{f^{n} z=x \\
z \notin Y, \ldots, f^{n} z \notin Y}}, \quad\left(B_{s, n} v\right)(x)=\sum_{\substack{f^{n} z=x \\
z \notin Y, \ldots, f^{n-1} z \notin Y ; f^{n} z \in Y}},
\end{aligned}
$$

and we have suppressed the summands $g_{n}(z) e^{s h_{n}(z)} v(z)$. Viewing these as operators $L_{s}: F_{\theta}(\Delta) \rightarrow L^{1}(\Delta), T_{s, n}: F_{\theta}(Y) \rightarrow L^{\infty}(Y), A_{s, n}: L^{\infty}(Y) \rightarrow L^{1}(\Delta), B_{s, n}:$ $F_{\theta}(\Delta) \rightarrow F_{\theta}(Y), E_{s, n}: L^{\infty}(\Delta) \rightarrow L^{1}(\Delta)$ (with the \|\|$_{b}$ norm on $F_{\theta}(Y)$ and $\left.F_{\theta}(\Delta)\right)$, we can write

$$
\left\|L_{s}^{n}\right\| \leq \sum_{i+j+k=n}\left\|A_{s, i}\right\|\left\|T_{s, j}\right\|\left\|B_{s, k}\right\|+\left\|E_{s, n}\right\| .
$$

We claim that

$$
\left\|A_{s, n}\right\| \leq C_{16} \gamma_{5}^{n}, \quad\left\|B_{s, n}\right\| \leq C_{16} \gamma_{5}^{n}, \quad\left\|E_{s, n}\right\| \leq C_{16} \gamma_{5}^{n} .
$$

Since $\left\|T_{s, n}\right\| \leq C|b|^{\alpha} e^{-n \delta|b|^{-\alpha}}$, the result follows from elementary facts about convolutions of sequences. (If $u_{n}=O\left(\delta^{n}\right), v_{n}=O\left(\epsilon^{n}\right)$, then $(u \star v)_{n}=O\left(\delta^{n}\right)$ when $\delta>\epsilon$ and $(u \star v)_{n}=O\left(n \delta^{n}\right)$ when $\delta=\epsilon$.)

It remains to verify estimates (4.1). Note that the support of $A_{s, n} v$ is contained in level $n$ of the tower and has measure at most $\sum_{r(j)>n} \mu\left(Y_{j}\right)=\sum_{k>n} \mu\left(Z_{k}\right)$ where $\mu\left(Z_{k}\right)=O\left(\gamma_{1}^{k}\right)$. For $x$ in level $n$, we have $\left(A_{s, n} v\right)(x)=e^{s h_{n}(z)} v(z)$ where 
$z$ is the unique point in $Y$ with $f^{n} z=x$, and so $\left|A_{s, n} v\right|_{\infty} \leq e^{n \epsilon|h|_{\infty}}|v|_{\infty}$. Hence $\left|A_{s, n} v\right|_{1} \leq C_{16} \gamma_{5}^{n}|v|_{\infty}$. Similarly,

$$
\left\|E_{s, n}\right\| \leq e^{n \epsilon|h|_{\infty}} \sum_{\substack{r(j)>n \\ n<\ell<r(j)}} \mu\left(\Delta_{j, \ell}\right) \leq e^{n \epsilon|h|_{\infty}} \sum_{r(j)>n} r(j) \mu\left(Y_{j}\right) \leq C_{16} \gamma_{5}^{n} .
$$

Finally, if $v: \Delta \rightarrow \mathbb{R}$ and $x \in Y$, then $\left(B_{s, n} v\right)(x)=\sum_{r(j)>n} e^{p\left(z_{j}\right)} e^{s h_{n}\left(z_{j}\right)} v\left(z_{j}\right)$ where $z_{j}$ is the unique preimage of $x$ in $\Delta_{j, r(j)-n}$. Hence the estimate is obtained in the same way as was done for $\left\|R_{s, n}\right\|_{b}$ in proving Propositions 3.9. (c) and 4.1.

Again, decay of correlations is a standard consequence of Lemma 4.4 Define the suspension semiflow $f_{t}: \Delta^{h} \rightarrow \Delta^{h}$ and the space of observables $F_{m, \theta}\left(\Delta^{h}\right)$ as before.

Proposition 4.5. Let $f: \Delta \rightarrow \Delta$ be a tower map with exponential tails, and let $h: \Delta \rightarrow \mathbb{R}^{+}$be a uniformly Lipschitz roof function. Suppose that the suspension flow $f_{t}: \Delta^{h} \rightarrow \Delta^{h}$ is mixing and that the estimates on $L_{s}^{n}$ in Lemma 4.4 are valid.

Then $f_{t}: \Delta^{h} \rightarrow \Delta^{h}$ is rapid mixing: for any $n \geq 1$, there exists $m \geq 1$ and $C \geq 1$ such that $\left|\rho_{v, w}(t)\right| \leq C\|v\|_{m, \theta}|w|_{\infty} t^{-n}$ for all $v \in F_{m, \theta}\left(\Delta^{h}\right), w \in L^{\infty}\left(\Delta^{h}\right)$, and $t>0$.

Proof. By assumption, $\sum_{n \geq 1}\left|L_{s}^{n} v\right|_{1} \leq C|b|^{\alpha}\left(1-e^{-\delta|b|^{-\alpha}}\right)^{-1}\|v\|_{b}$ for all $s=a+i b$ with $|b|>1$ and $|a| \leq \epsilon|b|^{-\alpha}$. But $\left(1-e^{-x}\right)^{-1} \leq 2 / x$ for $x$ small, and $\|v\|_{b} \leq\|v\|_{\theta}$, so there exists $C^{\prime}$ such that $\sum_{n>1}\left|L_{s}^{n} v\right|_{1} \leq C^{\prime}|b|^{2 \alpha}\|v\|_{\theta}$ for all such $s$. The remainder of the proof mimics the proof of Proposition 3.6. In fact the estimates are simpler since $h$ is uniformly Lipschitz, and there is no need to approximate $v$ by $\tilde{v}$.

4.3. Proof of Theorem 2.3. Let $T: X \rightarrow X$ be a nonuniformly expanding map as defined in Section 2, with partition $\bigcup_{j \geq 1} Y_{j}=Y$, return time function $r: Y \rightarrow \mathbb{Z}^{+}$, and ergodic invariant measure $m$. Note that the induced map $F: Y \rightarrow Y$ given by $F(y)=T^{r(y)}(y)$ is Gibbs-Markov. We build a tower $\Delta=\{(y, \ell): y \in Y, \ell=$ $0, \ldots, r(y)-1\}$, so $\Delta$ is partitioned into subsets $\Delta_{j, \ell}=Y_{j} \times\{\ell\}$ where $j \geq 1$ and $\ell=0, \ldots, r(j)-1$. Define the tower map $f: \Delta \rightarrow \Delta$ by setting $f(y, \ell)=(y, \ell+1)$ for $0 \leq \ell<r(y)-1$ and $f(y, r(y)-1)=(F y, 0)$. Let $\left.\mu\right|_{\Delta_{j, \ell}}=\left.m\right|_{Y_{j}} /|r|_{1}$, defining an ergodic $f$-invariant probability measure $\mu$ on $\Delta$.

The definition of a nonuniformly expanding map introduced the constants $\lambda>1$ and $\eta \in(0,1)$. Setting $\theta=1 / \lambda^{\eta} \in(0,1)$, we define the separation time $s$ and metric $d_{\theta}$ on $\Delta$ as in Subsection 4.1. Define the measure-preserving projection $\pi: \Delta \rightarrow X$ by $\pi(y, \ell)=T^{\ell} y$. This is a semiconjugacy between $f: \Delta \rightarrow \Delta$ and $T: X \rightarrow X$.

Proposition 4.6. $d(\pi p, \pi q)^{\eta} \leq C_{17} d_{\theta}(p, q)$ for all $p, q \in \Delta$.

Proof. If $p, q$ lie in distinct partition elements, then there is nothing to prove, so suppose that $p=(x, \ell), q=(y, \ell)$. Then $d(\pi p, \pi q)=d\left(T^{\ell} x, T^{\ell} y\right) \leq C d(F x, F y)$ by definition of $\pi$ and property (3) of $T$. By property $(2), d(F x, F y) \leq \lambda^{-s(F x, F y)} \leq$ $\lambda\left(\theta^{1 / \eta}\right)^{s(x, y)}=\lambda d_{\theta}(p, q)^{1 / \eta}$.

Let $h: X \rightarrow \mathbb{R}^{+}$be a roof function satisfying property (5) in Section 2 and define $\tilde{h}=h \circ \pi: \Delta \rightarrow \mathbb{R}^{+}$. It follows from Proposition 4.6 that $\tilde{h} \in F_{\theta}(\Delta)$. Define the suspension flows $T_{t}: X^{h} \rightarrow X^{h}$ and $f_{t}: \Delta^{\tilde{h}} \rightarrow \Delta^{\tilde{h}}$ with ergodic measures $m \times \ell /|h|_{1}$ and $\mu \times \ell /|h|_{1}$. Note that $\pi(p, u)=(\pi p, u)$ defines a measure-preserving 
semiconjugacy between the suspension flows on $\Delta^{\tilde{h}}$ and $X^{h}$. Moreover, if $v \in$ $C^{m, \eta}\left(X^{h}\right)$, then $\tilde{v}=v \circ \pi \in F_{m, \theta}(\Delta)$. Indeed $\|\tilde{v}\|_{m, \theta} \leq C_{17}\|v\|_{m, \eta}$.

Proof of Theorem 2.3. It is immediate from the discussion above that rapid mixing for $f_{t}: \Delta^{\tilde{h}} \rightarrow \Delta^{\tilde{h}}$ implies rapid mixing for $T_{t}: X^{h} \rightarrow X^{h}$. Hence we may suppose that $f_{t}$ is not rapid mixing. It follows from the results in Subsection 4.2 that the estimate $\left\|\left(I-R_{s, z}\right)^{-1}\right\|_{b} \leq C|b|^{\alpha}$ in Lemma 4.3 is violated. By Lemma 3.14, we conclude that $M_{b, \omega}$ has approximate eigenfunctions when restricted to any subsystem $Z_{0}$ of the Gibbs-Markov map $F: Y \rightarrow Y$. If $y \in Z_{0}$ is a periodic point for $F: Y \rightarrow Y$ of period $q$, then we define $d(y)=r_{q}(y)$ and $\tau(y)=H_{q}(y)$ where $H(x)=h_{r(x)}(x)$. Observe that $\left(M_{b, \omega}^{q n} u\right)(y)=e^{-i b n \tau} e^{-i \omega n d} u(y)$ for all $u: Y \rightarrow \mathbb{R}$, $n \geq 1$. Hence, the approximate eigenfunction criterion reduces to the estimate $\left|e^{i\left[b_{k} n_{k} \tau+\omega_{k} n_{k} d+q \varphi_{k}\right]}-1\right| \leq C q\left|b_{k}\right|^{-\alpha}$ for the triple $(\tau, d, q)$.

\section{RAPID MIXING FOR NONUNIFORMLY HYPERBOLIC FLOWS}

In this section we prove Theorem 2.6. In Subsection 5.1 we include the necessary background material and notation from Young 35] on nonuniformly hyperbolic maps and towers. In Subsection 5.2, we use approximation arguments to reduce the nonuniformly hyperbolic case to the nonuniformly expanding case studied in Section 4 .

5.1. Background on nonuniformly hyperbolic maps. Let $T: M \rightarrow M$ be a nonuniformly hyperbolic map in the sense of Young [35]. As described in Section 2.2, there is a partition $\left\{Y_{j}\right\}$ of $Y \subset M$ with return time function $r: Y \rightarrow \mathbb{Z}^{+}$, constant on partition elements $\left\{Y_{j}\right\}$, and induced return map $F: Y \rightarrow Y$ given by $F(y)=$ $T^{r(y)}(y)$. The hypotheses in Young [35] guarantee the existence of an ergodic $T$ invariant probability measure $m$ that is an SRB measure.

Let $\Delta=\{(y, \ell): y \in Y, \ell=0, \ldots, r(y)-1\}$ and define the tower map $f: \Delta \rightarrow \Delta$ by setting $f(y, \ell)=(y, \ell+1)$ for $0 \leq \ell<r(y)-1$ and $f(y, r(y)-1)=(F y, 0)$. The projection $\pi: \Delta \rightarrow M$ given by $\pi(x, \ell)=T^{\ell} x$ is a semiconjugacy between $f: \Delta \rightarrow \Delta$ and $T: M \rightarrow M$.

The subset $Y$ is covered by families of stable disks $\left\{W^{s}(x), x \in Y\right\}$ and unstable disks $\left\{W^{u}(x), x \in Y\right\}$ such that each stable disk intersects each unstable disk in exactly one point. For $p=(x, \ell), q=(y, \ell) \in \Delta$, we write $q \in W^{s}(p)$ if $y \in W^{s}(x)$ (and $q \in W^{u}(p)$ if $y \in W^{u}(x)$ ).

Quotienting out the stable directions, we obtain the quotient maps $\bar{f}: \bar{\Delta} \rightarrow \bar{\Delta}$ and $\bar{F}: \bar{Y} \rightarrow \bar{Y}$. The hypotheses in [35] guarantee that:

Proposition 5.1. The quotient tower map $\bar{f}: \bar{\Delta} \rightarrow \bar{\Delta}$ is a nonuniformly expanding tower map as defined in Section 4. In particular, there are measures $\bar{\mu}$ and $\bar{\mu} \times \nu /|r|_{1}$ on $\bar{Y}$ and $\bar{\Delta}$ respectively, such that $\bar{F}: \bar{Y} \rightarrow \bar{Y}$ is Gibbs-Markov with respect to the quotient partition $\left\{\bar{Y}_{j}\right\}$. Moreover, there is an f-invariant measure $\mu$ on $\Delta$ such that the natural projection $\bar{\pi}: \Delta \rightarrow \bar{\Delta}$ and the projection $\pi: \Delta \rightarrow M$ are measurepreserving semiconjugacies.

In Section 4, we defined a separation time $s: \bar{\Delta} \times \bar{\Delta} \rightarrow \mathbb{N}$ defined relative to returns under $\bar{F}$ to the partition $\left\{\bar{Y}_{j}\right\}$. (This is the separation time used in [15, 36]). For $\theta \in(0,1)$, we again define the metric $d_{\theta}(p, q)=\theta^{s(p, q)}$.

We now introduce a new separation time $s_{1}: \Delta \times \Delta \rightarrow \mathbb{N}$ defined in terms of $f$. (This plays the same role as the separation time $s$ in [35, but it is different 
from the separation times in [35, 36.) As in Section 4.1, the quotient tower map $\bar{f}: \bar{\Delta} \rightarrow \bar{\Delta}$ is Markov with respect to the partition $\left\{\bar{\Delta}_{j, \ell}\right\}$ where $\bar{\Delta}_{j, \ell}=\bar{Y}_{j} \times\{\ell\}$ for $j \geq 1$ and $\ell=0, \ldots, r(j)-1$. Define $s_{1}: \bar{\Delta} \times \bar{\Delta} \rightarrow \mathbb{N}$ by setting $s_{1}(\bar{p}, \bar{q})$ to be the least integer $n$ such that $\bar{f}^{n} \bar{p}$ and $\bar{f}^{n} \bar{q}$ lie in distinct partition elements $\bar{\Delta}_{j, \ell}$. Define $s_{1}: \Delta \times \Delta \rightarrow \mathbb{N}$ by setting $s_{1}(p, q)=s_{1}(\bar{\pi} p, \bar{\pi} q)$. Note that the separation times $s_{1} \geq s$ are defined on both $\bar{\Delta}$ and $\Delta$, but the metric $d_{\theta}$ is defined only on $\bar{\Delta}$ and always in terms of $s$.

Proposition 5.2. (a) If $q \in W^{s}(p)$, then $d\left(\pi f^{n} p, \pi f^{n} q\right) \leq C_{18} \gamma_{6}^{n}$ for all $n \geq 1$.

(b) If $q \in W^{u}(p)$, then $d\left(\pi f^{n} p, \pi f^{n} q\right) \leq C_{18} \gamma_{6}^{s_{1}(p, q)-n}$ for $0 \leq n<s_{1}(p, q)$.

Proof. This follows from the set-up in [35], with some additional care required due to the different definition of separation time. Write $p=(x, \ell), q=(y, \ell) \in \Delta$. Then $d\left(\pi f^{n} p, \pi f^{n} q\right)=d\left(T^{n+\ell} x, T^{n+\ell} y\right)$. If $q \in W^{s}(p)$, then $y \in W^{s}(x)$, and it follows from [35, P3] that

$$
d\left(\pi f^{n} p, \pi f^{n} q\right)=d\left(T^{n+\ell} x, T^{n+\ell} y\right) \leq C_{18} \gamma_{6}^{n+\ell} \leq C_{18} \gamma_{6}^{n} .
$$

If $q \in W^{u}(p)$, then $y \in W^{u}(x)$, and it follows from [35, P4a] that

$$
d\left(\pi f^{n} p, \pi f^{n} q\right)=d\left(T^{n+\ell} x, T^{n+\ell} y\right) \leq C_{18} \gamma_{6}^{s_{0}(x, y)-(n+\ell)}
$$

where $s_{0}$ is the separation time in 35. Note that $s_{0}(x, y) \geq s_{1}((x, 0),(y, 0))$, and hence $s_{1}(p, q)=s_{1}((x, \ell),(y, \ell))=s_{1}((x, 0),(y, 0))-\ell \leq s_{0}(x, y)-\ell$. The result follows.

Corollary 5.3. $d\left(T^{n} \pi p, T^{n} \pi q\right) \leq 2 C_{18} \gamma_{6}^{\min \left\{n, s_{1}(p, q)-n\right\}}$ for all $p, q \in \Delta, 0 \leq n \leq$ $s_{1}(p, q)$.

Proof. Define $z=W^{s}(p) \cap W^{s}(q)$. By Proposition 5.2(a), $d\left(\pi f^{n} p, \pi f^{n} z\right) \leq C_{18} \gamma_{6}^{n}$. Moreover, $s_{1}(z, q)=s_{1}(p, q)$. By Proposition $5.2(\mathrm{~b}), d\left(\pi f^{n} z, \pi f^{n} q\right) \leq C_{18} \gamma_{6}^{s_{1}(p, q)-n}$.

5.2. Proof of Theorem 2.6. We continue to assume that $T: M \rightarrow M$ is a nonuniformly hyperbolic map, modelled by a Young tower $f: \Delta \rightarrow \Delta$ as in Subsection 5.1 . We have the measure-preserving semiconjugacy $\pi: \Delta \rightarrow M$.

Let $h: M \rightarrow \mathbb{R}^{+}$be an $\eta$-Hölder roof function with associated suspension flow $T_{t}: M^{h} \rightarrow M^{h}$. Define $\tilde{h}=h \circ \pi$ with suspension flow $f_{t}: \Delta^{\tilde{h}} \rightarrow \Delta^{\tilde{h}}$. The projection $\pi: \Delta^{\tilde{h}} \rightarrow M^{h}$ defined by $\pi(p, u)=(\pi p, u)$ is a measure-preserving conjugacy.

Suppose that $v, w \in C^{\eta}\left(M^{h}\right)$ and let $\tilde{v}=v \circ \pi, \tilde{w}=w \circ \pi$. It suffices to prove decay of correlations for the observations $\tilde{v}, \tilde{w}: \Delta^{\tilde{h}} \rightarrow \mathbb{R}$. As in previous sections, the significant part of the Laplace transform of $\rho$ has the form $\hat{\rho}(s)=\sum_{n \geq 0} \int_{\Delta} e^{-s \tilde{h}_{n}} v_{s} w_{s} \circ f^{n} d \mu$ where $v_{s}(p)=\int_{0}^{\tilde{h}(p)} e^{s u} \tilde{v}(p, u) d u$ and $w_{s}(p)=$ $\int_{0}^{\tilde{h}(p)} e^{-s u} \tilde{w}(p, u) d u$. Superpolynomial decay of correlations follows from an estimate of the form

$$
|\hat{\rho}(s)| \leq C|b|^{\alpha}\|v\|_{\eta}\|w\|_{\eta}
$$

for $s=a+i b$ with $|b|>1$ and $|a| \leq \epsilon|b|^{-\alpha}$.

It remains to establish (5.1). The first step [33, 6] is to write $\tilde{h}$ as a coboundary plus a roof function that "depends only on future coordinates". We adapt a result of [24, Lemma 3.2] formulated for the nonuniformly hyperbolic setting. 
Lemma 5.4. There exist functions $\bar{h}, \chi: \Delta \rightarrow \mathbb{R}$ such that

(i) $\tilde{h}=\bar{h}+\chi-\chi \circ f$,

(ii) $\chi \in L^{\infty}(\Delta)$,

(iii) if $s_{1}(p, q) \geq 3 k$, then $\left|\chi\left(f^{k} p\right)-\chi\left(f^{k} q\right)\right| \leq C_{19} \gamma_{7}^{k}$, where $\gamma_{7}=\gamma_{6}^{\eta}$,

(iv) $\bar{h}(p)=\bar{h}(q)$ for all $p \in W^{s}(q)$,

(v) $\bar{h}: \bar{\Delta} \rightarrow \mathbb{R}$ is Lipschitz with respect to the metric $d_{\theta}$, for $\theta=\gamma_{7}^{1 / 2}$.

Proof. Fix an unstable disk $W^{u}$. Given $p=(x, \ell) \in \Delta$, define $\hat{p}=(\hat{x}, \ell)$ where $\hat{x}$ is the unique point in $W^{s}(x) \cap W^{u}$. Define

$$
\chi(p)=\sum_{j=0}^{\infty} h\left(\pi f^{j} p\right)-h\left(\pi f^{j} \hat{p}\right) .
$$

It follows from Proposition 5.2 (a) that

$$
\begin{aligned}
|\chi(p)| & \leq\left.\sum_{j=0}^{\infty}\left|h\left(\pi f^{j} p\right)-h\left(\pi f^{j} \hat{p}\right) \leq\right| h\right|_{\eta} \sum_{j=0}^{\infty} d\left(T^{j} \pi p, T^{j} \pi \hat{p}\right)^{\eta} \\
& \leq|h|_{\eta} C_{18}^{\eta} \sum_{j=0}^{\infty} \gamma_{7}^{j}=|h|_{\eta} C_{18}^{\eta}\left(1-\gamma_{7}\right)^{-1},
\end{aligned}
$$

proving (ii).

Next, we note that to estimate $\left|\chi\left(z_{1}\right)-\chi\left(z_{2}\right)\right|$, it suffices to estimate the four terms

$$
\begin{array}{ll}
\sum_{j=0}^{k-1}\left|h\left(\pi f^{j} z_{1}\right)-h\left(\pi f^{j} z_{2}\right)\right|, & \sum_{j=0}^{k-1}\left|h\left(\pi f^{j} \hat{z_{1}}\right)-h\left(\pi f^{j} \hat{z_{2}}\right)\right|, \\
\sum_{j=k}^{\infty}\left|h\left(\pi f^{j} z_{1}\right)-h\left(\pi f^{j} \hat{z_{1}}\right)\right|, & \sum_{j=k}^{\infty}\left|h\left(\pi f^{j} z_{2}\right)-h\left(\pi f^{j} \hat{z_{2}}\right)\right| .
\end{array}
$$

The computation used to prove (ii) shows that the third and fourth terms are dominated by $C_{19} \gamma_{7}^{k}$ for all $z_{1}, z_{2} \in \Delta$. Hence, to prove (iii) it suffices to estimate the first and second terms with $z_{1}=f^{k} p, z_{2}=f^{k} q$, where $s_{1}(p, q) \geq 3 k$. The first term is dominated by $|h|_{\eta} \sum_{j=0}^{k-1} d\left(T^{j+k} \pi p, T^{j+k} \pi q\right)^{\eta}$. By Corollary 5.3, we obtain the estimate $C_{20} \sum_{j=0}^{k-1} \gamma_{7}^{\min \left(j+k, s_{1}(p, q)-j-k\right)} \leq C_{21} \gamma_{7}^{k}$ as required. Similarly for the second term, completing the proof of (iii).

Define $\bar{h}=h \circ \pi-\chi+\chi \circ f$. Then $\bar{h}(p)=\sum_{j=0}^{\infty} h\left(\pi f^{j} \hat{p}\right)-h\left(\pi f^{j} \widehat{f p}\right)$ depends only upon future coordinates. It remains to check that $\bar{h}$ is Lipschitz with respect to the metric $d_{\theta}$. In fact, we show that $|\bar{h}(p)-\bar{h}(q)| \leq C_{22} \theta^{s_{1}(p, q)}$. Let $p, q \in \Delta$ with $s_{1}(p, q) \geq 2 k$. There exist $\bar{p}^{\prime} \in \bar{f}^{-k} \bar{p}, \bar{q}^{\prime} \in \bar{f}^{-k} \bar{q}$ with $s_{1}\left(p^{\prime}, q^{\prime}\right) \geq 3 k$. By (i), (iii) and the Hölder continuity of $h$, we have that $|\bar{h}(p)-\bar{h}(q)|=\left|\bar{h}\left(f^{k} p^{\prime}\right)-\bar{h}\left(f^{k} q^{\prime}\right)\right| \leq C_{22} \gamma_{7}^{k}$ as required.

By Lemma 5.4, we can write $\hat{\rho}(s)=\sum_{n \geq 0} \int_{\Delta} e^{-s \bar{h}_{n}}\left(e^{-s \chi} v_{s}\right)\left(e^{s \chi} w_{s}\right) \circ f^{n} d \mu$. The next step is to approximate $e^{-s \chi} v_{s}$ and $e^{s \chi} w_{s}$ by functions that "depend only on finitely many coordinates".

For $k \geq 1$, define $v_{s, k}(p)=\inf \left\{\left(e^{-s \chi} v_{s}\right)\left(f^{k} q\right): s_{1}(p, q) \geq 3 k\right\}$. 
Lemma 5.5. The function $v_{s, k}: \Delta \rightarrow \mathbb{R}$ lies in $L^{\infty}(\Delta)$ and projects down to a Lipschitz observation $\overline{v_{s, k}}: \bar{\Delta} \rightarrow \mathbb{R}$. Moreover, within the region $s=a+i b,|a| \leq$ $1,|b| \geq 1$

(a) $\left|\overline{v_{s, k}}\right|_{\infty}=\left|v_{s, k}\right|_{\infty} \leq e^{|\chi|_{\infty}}\left|v_{s}\right|_{\infty} \leq C_{23}|\tilde{v}|_{\infty}=C_{23}|v|_{\infty}$.

(b) $\left|\overline{v_{s, k}}\right|_{\theta} \leq 2 C_{23} \theta^{-3 k}|v|_{\infty}$.

(c) $\left|\left(e^{-s \chi} v_{s}\right) \circ f^{k}-v_{s, k}\right|_{\infty} \leq C_{24}|b|\|v\|_{\eta} \gamma_{7}^{k}$.

Proof. Define $\mathcal{M}(\bar{p})=\left\{\bar{q} \in \bar{\Delta}: s_{1}(\bar{p}, \bar{q}) \geq 3 k\right\}$. It is clear that $\{\mathcal{M}(\bar{p})\}$ defines a measurable partition of $\bar{\Delta}$ and so $\left\{\bar{\pi}^{-1} \mathcal{M}(\bar{p})\right\}$ is a measurable partition of $\Delta$. By definition $v_{s, k}$ is constant on such partition elements and hence is measurable.

If $s_{1}(p, q) \geq 3 k$, then $v_{s, k}(p)=v_{s, k}(q)$. In particular, $\overline{v_{s, k}}: \bar{\Delta} \rightarrow \mathbb{R}$ is welldefined. Part (a) is immediate. Moreover, $\left|\overline{v_{s, k}}\right|_{\theta}=\sup _{p \neq q}\left|v_{s, k}(p)-v_{s, k}(q)\right| / \theta^{s(p, q)}$ where $s$ is the separation time defined in terms of $F$. If $s(p, q) \geq 3 k$, then certainly $s_{1}(p, q) \geq 3 k$ and so $v_{s, k}(p)-v_{s, k}(q)=0$. Hence, we can restrict to pairs $p, q$ with $s(p, q) \leq 3 k$. It follows that $\left|\overline{v_{s, k}}\right|_{\theta} \leq 2\left|v_{s, k}\right|_{\infty} \theta^{-3 k}$ proving part (b).

Note that $\left|\left(e^{-s \chi} v_{s}\right) \circ f^{k}-v_{s, k}\right|_{\infty} \leq \sup _{s_{1}(p, q) \geq 3 k}\left|\left(e^{-s \chi} v_{s}\right)\left(f^{k} p\right)-\left(e^{-s \chi} v_{s}\right)\left(f^{k} q\right)\right|$. By Lemma 5.4(iii), $\left|e^{-s \chi\left(f^{k} p\right)}-e^{-s \chi\left(f^{k} q\right)}\right| \leq C_{25}|b| \gamma_{7}^{k}$. Also,

$$
\begin{aligned}
\mid v_{s}(p) & -v_{s}(q)|\leq| \int_{\tilde{h}(q)}^{\tilde{h}(p)} e^{s u} \tilde{v}(p, u) d u|+| \int_{0}^{\tilde{h}(q)} e^{s u}(\tilde{v}(p, u)-\tilde{v}(q, u)) d u \mid \\
& \leq C_{26}\left\{|\tilde{h}(p)-\tilde{h}(q)||\tilde{v}|_{\infty}+|\tilde{v}(p, u)-\tilde{v}(q, u)|\right\} \\
& \leq C_{26}\left\{|h(\pi p)-h(\pi q)||v|_{\infty}+|v(\pi p, u)-v(\pi q, u)|\right\} \leq C_{27}\|v\|_{\eta} d(\pi p, \pi q)^{\eta},
\end{aligned}
$$

and so part (c) follows from Corollary 5.3 .

Write $\int_{\Delta} e^{-s \bar{h}_{n}}\left(e^{-s \chi} v_{s}\right)\left(e^{s \chi} w_{s}\right) \circ f^{n} d \mu=\int_{\Delta} e^{-s \bar{h}_{n} \circ f^{k}}\left(e^{-s \chi} v_{s}\right) \circ f^{k}\left(e^{s \chi} w_{s}\right) \circ f^{k} \circ$ $f^{n} d \mu=I_{1}+I_{2}+I_{3}$, where

$$
\begin{aligned}
& I_{1}=\int_{\Delta} e^{-s \bar{h}_{n} \circ f^{k}}\left(e^{-s \chi} v_{s}\right) \circ f^{k}\left(\left(e^{s \chi} w_{s}\right) \circ f^{k}-w_{s, k}\right) \circ f^{n} d \mu, \\
& I_{2}=\int_{\Delta} e^{-s \bar{h}_{n} \circ f^{k}}\left(\left(e^{-s \chi} v_{s}\right) \circ f^{k}-v_{s, k}\right) w_{s, k} \circ f^{n} d \mu, \\
& I_{3}=\int_{\Delta} e^{-s \bar{h}_{n} \circ f^{k}} v_{s, k} w_{s, k} \circ f^{n} d \mu .
\end{aligned}
$$

By Lemma 5.5 ,

$$
\left|I_{1}\right| \leq e^{n|a||h|_{\infty}}\left|v_{s}\right|_{\infty}\left|\left(e^{s \chi} w_{s}\right) \circ f^{k}-w_{s, k}\right|_{\infty} \leq C_{28}|b| e^{n|a||h|_{\infty}}|v|_{\infty}\|w\|_{\eta} \gamma_{7}^{k},
$$

and similarly $\left|I_{2}\right| \leq C_{28}|b| e^{n|a||h|_{\infty}}\|v\|_{\eta}|w|_{\infty} \gamma_{7}^{k}$. The integrand in $I_{3}$ projects down to $\bar{\Delta}$ and $\bar{h}_{n} \circ \bar{f}^{k}=\bar{h}_{n}+\bar{h}_{k} \circ \bar{f}^{n}-\bar{h}_{k}$, so we obtain

$$
I_{3}=\int_{\bar{\Delta}} e^{-s \bar{h}_{n}}\left[e^{s \bar{h}_{k}} \overline{v_{s, k}}\right]\left[e^{-s \bar{h}_{k}} \overline{w_{s, k}}\right] \circ \bar{f}^{n} d \mu=\int_{\bar{\Delta}} L_{-s}^{n}\left[e^{s \bar{h}_{k}} \overline{v_{s, k}}\right]\left[e^{-s \bar{h}_{k}} \overline{w_{s, k}}\right] d \mu .
$$

Restricting to the region $s=a+i b$ with $|b|>1$ and $|a| \leq \epsilon|b|^{-\alpha}$,

$$
\left|I_{1}\right|,\left|I_{2}\right| \leq C_{28}|b| \gamma_{7}^{k} e^{n \epsilon|b|^{-\alpha}|h|_{\infty}}\|v\|_{\eta}\|w\|_{\eta} .
$$

By Lemma 4.4. $\left|L_{-s}^{n} u\right|_{1} \leq C|b|^{\alpha} e^{-n \delta|b|^{-\alpha}}\|u\|_{\theta}$ for $u \in F_{\theta}(\bar{\Delta})$. Hence,

$$
\begin{aligned}
\left|I_{3}\right| & \leq C|b|^{\alpha} e^{-n \delta|b|^{-\alpha}}\left\|e^{s \bar{h}_{k}}\right\|_{\theta}\left\|\overline{v_{s, k}}\right\|_{\theta}\left|e^{-s h_{k}}\right|_{\infty}\left|\overline{w_{s, k}}\right|_{\infty} \\
& \leq C|b|^{\alpha+1} e^{-n \delta|b|^{-\alpha}} \theta^{-4 k} e^{2 k|h|_{\infty}}|v|_{\infty}|w|_{\infty} .
\end{aligned}
$$

Choose $k=k(b, n)$ such that $\left(e^{2|h|_{\infty}} \theta^{-4}\right)^{k} \sim e^{n \delta|b|^{-\alpha} / 2}$. Then there exists $\delta^{\prime}>$ 0 (depending on $\gamma_{7}$ and $\theta$ ) such that $I_{1}, I_{2}=O\left(e^{-\left(\delta^{\prime}-\epsilon\right) n|b|^{-\alpha}|h|_{\infty}}|b|\right)$ and $I_{3}=$ 
$O\left(e^{-n \delta|b|^{-\alpha} / 2}|b|^{\alpha+1}\right)$. Choosing $\epsilon$ small enough, we obtain a new $\delta^{\prime}>0$ such that

$$
\left|\int_{\Delta} e^{-s \bar{h}_{n}}\left(e^{-s \chi} v_{s}\right)\left(e^{s \chi} w_{s}\right) \circ f^{n} d \mu\right| \leq C e^{-n \delta^{\prime}|b|^{-\alpha}}|b|^{\alpha+1}\|v\|_{\eta}\|w\|_{\eta} .
$$

Summing over $n$, and using the fact that $\left(1-e^{-x}\right)^{-1} \leq 2 / x$ for $x>0$ small, we obtain $|\hat{\rho}(s)| \leq C^{\prime}\|v\|_{\eta}\|w\|_{\eta}|b|^{2 \alpha+1}$ as required.

\section{REFERENCES}

1. J. Aaronson. An Introduction to Infinite Ergodic Theory. Math. Surveys and Monographs 50, Amer. Math. Soc., 1997. MR.1450400 (99d:28025)

2. M. Benedicks and L. Carleson. The dynamics of the Hénon map. Ann. of Math. 133 (1991) 73-169. MR1087346 (92d:58116)

3. M. Benedicks and L.-S. Young. Sinai-Bowen-Ruelle measures for certain Hénon maps. Invent. Math. 112 (1993) 541-576. MR.1218323 (94e:58074)

4. M. Benedicks and L.-S. Young. Markov extensions and decay of correlations for certain Hénon maps. Astérisque (2000) no. 261, 13-56. MR1755436 (2001g:37030)

5. R. Bowen. Symbolic dynamics for hyperbolic flows. Amer. J. Math. 95 (1973) 429-460. MR0339281 (49:4041)

6. R. Bowen. Equilibrium States and the Ergodic Theory of Anosov Diffeomorphisms, Lecture Notes in Math. 470, Springer, Berlin, 1975. MR0442989 (56:1364)

7. R. Bowen and D. Ruelle. The ergodic theory of Axiom A flows. Invent. Math. 29 (1975) 181-202. MR0380889 (52:1786)

8. M. I. Brin. Topological transitivity of a certain class of dynamical systems, and flows of frames on manifolds of negative curvature. Func. Anal. Appl. 9 (1975) 9-19. MR0370660 (51:6886)

9. M. I. Brin. The topology of group extensions of $C$-systems. Mat. Zametki 18 (1975) 453-465. MR0394764 (52:15563)

10. H. Bruin, M. Holland and I. Melbourne. Subexponential decay of correlations for compact group extensions of nonuniformly expanding systems. Ergod. Th. \& Dynam. Sys. 25 (2005) 1719-1738. MR2183290(2006i:37067)

11. L. A. Bunimovich, Y. G. Sină and N. I. Chernov. Statistical properties of two-dimensional hyperbolic billiards. Uspekhi Mat. Nauk 46 (1991) 43-92. MR.1138952 (92k:58151)

12. N. I. Chernov. Markov approximations and decay of correlations for Anosov flows. Ann. of Math. 147 (1998) 269-324. MR.1626741 (99d:58101)

13. N. I. Chernov. Decay of correlations and dispersing billiards. J. Statist. Phys. 94 (1999) 513556. MR.1675363 (2000j:37044)

14. N. I. Chernov. Sinai billiards under small external forces. Ann. Henri Poincaré 2 (2001) 197-236 MR 1832968 (2002c:37053)

15. N. Chernov and L. S. Young. Decay of correlations for Lorentz gases and hard balls. Hard ball systems and the Lorentz gas. Encyclopaedia Math. Sci. 101, Springer, Berlin, 2000, pp. 89120. MR1805327 (2001k:37046)

16. L. J. Díaz, J. Rocha and M. Viana. Strange attractors in saddle-node cycles: prevalence and globality. Invent. Math. 125 (1996) 37-74. MR1389960 (97h:58109)

17. D. Dolgopyat. On the decay of correlations in Anosov flows. Ann. of Math. 147 (1998) 357390. MR1626749 (99g:58073)

18. D. Dolgopyat. Prevalence of rapid mixing in hyperbolic flows. Ergod. Th. EG Dynam. Sys. 18 (1998) 1097-1114. MR1653299 (2000a:37014)

19. M. J. Field, I. Melbourne and A. Török. Stability of mixing and rapid mixing for hyperbolic flows. To appear in Ann. of Math.

20. S. Gouëzel. Sharp polynomial estimates for the decay of correlations. Israel J. Math. 139 (2004) 29-65. MR2041223 (2004m:37011)

21. S. Gouëzel. Berry-Esseen theorem and local limit theorem for non uniformly expanding maps. Ann. Inst. H. Poincaré Probab. Statist. 41 (2005) 997-1024. MR2172207

22. S. Gouëzel. Vitesse de décorrélation et théorèmes limites pour les applications non uniformément dilatantes. Ph.D. Thesis. Ecole Normale Supérieure, 2004.

23. C. Liverani. On contact Anosov flows. Ann. of Math. 159 (2004) 1275-1312. MR 2113022 (2005k:37048)

24. I. Melbourne and M. Nicol. Almost sure invariance principle for nonuniformly hyperbolic systems. Commun. Math. Phys. 260 (2005) 131-146. MR2175992(2006h:37047) 
25. I. Melbourne and A. Török. Central limit theorems and invariance principles for time-one maps of hyperbolic flows. Commun. Math. Phys. 229 (2002) 57-71. MR.1917674 (2003k:37012)

26. I. Melbourne and A. Török. Statistical limit theorems for suspension flows. Israel J. Math. 194 (2004) 191-210. MR2121540 (2006c:37005)

27. L. Mora and M. Viana. Abundance of strange attractors. Acta. Math. 171 (1993) 1-71. MR 1237897 (94k:58089)

28. M. Pollicott. On the rate of mixing of Axiom A flows. Invent. Math. 81 (1985) 413-426. MR0807065 (87i:58148)

29. M. Pollicott. On the rate of mixing of Axiom A attracting flows and a conjecture of Ruelle. Ergod. Th. \& Dynam. Sys. 19 (1999) 535-548. MR1685406 (2001d:37038)

30. D. Ruelle. Flows which do not exponentially mix. C. R. Acad. Sci. Paris 296 (1983) 191-194. MR0692974 (84h:58085)

31. O. M. Sarig. Subexponential decay of correlations. Invent. Math. 150 (2002) 629-653. MR $1946554(2004 \mathrm{e}: 37010)$

32. Y. G. Sina1̆. Dynamical systems with elastic reflections. Ergodic properties of dispersing billiards. Uspehi Mat. Nauk 25 (1970) 141-192. MR0274721 (43:481)

33. Y. G. Sină. Gibbs measures in ergodic theory. Russ. Math. Surv. 27 (1972) 21-70. MR0399421 $(53: 3265)$

34. M. Viana. Strange attractors in higher dimensions. Bol. Soc. Brasil. Mat. 24 (1993) 13-62. MR 1224299 (94k:58093)

35. L.-S. Young. Statistical properties of dynamical systems with some hyperbolicity. Ann. of Math. 147 (1998) 585-650. MR 1637655 (99h:58140)

36. L.-S. Young. Recurrence times and rates of mixing. Israel J. Math. 110 (1999) 153-188. MR:1750438 (2001j:37062)

Department of Mathematics and Statistics, University of Surrey, Guildford GU2 7XH, United KINGDOM

E-mail address: ism@math.uh.edu 